Supporting information for

\title{
Efficient Allylation of Aldehydes Promoted by Carboxylic Acids
}

\author{
Gui-long Li, Gang Zhao* \\ E-mail: Zhaog@mail.sioc.ac.cn
}

General experimental methods and data of the compounds of the allylation------S2-S9

NMR spectras of the compounds $-\mathrm{S} 10-\mathrm{S} 24$ 


\section{General:}

Allylic stannanes $\left(\mathbf{2 a},{ }^{1} \mathbf{2 b},{ }^{2}\right), 4^{3}$ were prepared according to the reported procedures. Tributyltin ester of $p$-nitro benzoic acid was prepared according to a similar procedure in literature. ${ }^{4}$ All carboxylic acids, aldehydes and solvent are commercially available and were used without further purification.

The solubility of carboxylic acids was obtained by the following procedure: the carboxylic acid $(0.2 \mathrm{~g})$ was added into acetonitrile $(4 \mathrm{~mL})$ at $20{ }^{\circ} \mathrm{C}$. The mixture was stirred for $24 \mathrm{~h}$ (no carboxylic acid was dissolved completely). Then the above clear solution $(2 \mathrm{~mL})$ was took out and concentrated under reduced pressure to provide the dissolved carboxylic acid.

1-phenyl-3-buten-1-ol (3a) ${ }^{5}{ }^{1} \mathrm{H}$ NMR $\left(300 \mathrm{MHz}, \mathrm{CDCl}_{3}\right): \delta 7.37-7.26(\mathrm{~m}, 5 \mathrm{H})$, 5.87-5.78(m, 1H), 5.14(t, 2H, $J=9.1,9.6 \mathrm{~Hz}), 4.73(\mathrm{t}, 1 \mathrm{H}, J=5.4,7.3 \mathrm{~Hz})$, 2.54-2.49(m, 2H), 2.03(s, 1H); IR (KBr, film): v 3383, 3076, 3030, 2978, 2906, 1642 , $1494,1455,1435,1048,916,700 \mathrm{~cm}^{-1}$.

1-(4-nitrophenyl)-3-buten-1-ol (3b) ${ }^{6}{ }^{1} \mathrm{H}$ NMR (300MHz, $\left.\mathrm{CDCl}_{3}\right): \delta 8.19(\mathrm{dd}, 2 \mathrm{H}$, $J=1.8,8.8 \mathrm{~Hz}), 7.52(\mathrm{~d}, 2 \mathrm{H}, J=8.5 \mathrm{~Hz}), 5.58-5.72(\mathrm{~m}, 1 \mathrm{H}), 5.23-5.17(\mathrm{~m}, 2 \mathrm{H}), 4.86(\mathrm{t}$, $1 \mathrm{H}, J=3.6 \mathrm{~Hz}), 2.61-2.38(\mathrm{~m}, 2 \mathrm{H}), 2.24(\mathrm{~d}, 1 \mathrm{H}, J=3.0 \mathrm{~Hz})$; IR (KBr, film): $v \quad 3416$, $3078,2911,1641,1604,1591,1347,1055 \mathrm{~cm}^{-1}$.

\section{1-(3-methoxyphenyl)-3-buten-1-ol $\quad(3 \mathbf{c})^{7} \quad{ }^{1} \mathrm{H} \quad \mathrm{NMR} \quad\left(300 \mathrm{MHz}, \quad \mathrm{CDCl}_{3}\right): \quad \delta$} 7.29-7.24(m, 1H), 6.94-6.92(m, 2H), 6.84-6.80(m, 1H), 5.88-5.75(m, 1H), 5.21-5.14(m, 1H), 4.73-4.70(m, 1H), 3.82(S, 3H), 2.53-2.48(m, 2H), 2.04(d, 1H, $J=$ $3.4 \mathrm{~Hz}$ ); IR (KBr, film): v 3415, 3075, 2936, 2836, 1641, 1602, 1586, 1489, 1264 $\mathrm{cm}^{-1}$ 
1-(2-furyl)-3-buten-1-ol (3e) ${ }^{8}{ }^{1} \mathrm{H}$ NMR $\left(300 \mathrm{MHz}, \mathrm{CDCl}_{3}\right): \delta 7.38(\mathrm{t}, 1 \mathrm{H}, J=1.1$ $\mathrm{Hz}), 6.33(\mathrm{t}, 1 \mathrm{H}, J=2.5 \mathrm{~Hz}), 6.25(\mathrm{t}, 1 \mathrm{H}, J=1.7 \mathrm{~Hz}), 5.86-5.75(\mathrm{~m}, 1 \mathrm{H}), 5.23-5.13(\mathrm{~m}$, 2H), 4.78(q, 1H, $J=5.9 \mathrm{~Hz}), 2.66-2.60(\mathrm{~m}, 2 \mathrm{H}), 2.02(\mathrm{~d}, 1 \mathrm{H}, J=4.9 \mathrm{~Hz})$; IR $(\mathrm{KBr}$, film): $v 3389,3078,2923,1642,1505,1434,1149,1011 \mathrm{~cm}^{-1}$.

1-(4-fluorophenyl)-3-buten-1-ol (3f) ${ }^{9}{ }^{1} \mathrm{H}$ NMR (300MHz, $\left.\mathrm{CDCl}_{3}\right): \delta 7.30(\mathrm{t}, 2 \mathrm{H}$, $J=7.0 \mathrm{~Hz}), 7.00(\mathrm{t}, 2 \mathrm{H}, J=8.7 \mathrm{~Hz}), 5.87-5.72(\mathrm{~m}, 1 \mathrm{H}), 5.20-5.13(\mathrm{~m}, 2 \mathrm{H}), 4.70(\mathrm{t}, 1 \mathrm{H}, J$ $=6.4 \mathrm{~Hz}), 2.51-2.45(\mathrm{~m}, 2 \mathrm{H}), 2.07(\mathrm{~s}, 1 \mathrm{H}) ; \mathrm{IR}(\mathrm{KBr}$, film): $v$ 3388, 3077, 2980, 2930, $1641,1605,1510,1224 \mathrm{~cm}^{-1}$.

1-(4-methoxyphenyl)-3-buten-1-ol (3i) ${ }^{10}{ }^{1} \mathrm{H}$ NMR $\left(300 \mathrm{MHz}, \mathrm{CDCl}_{3}\right): \delta$ 7.27(d, $2 \mathrm{H}, J=8.3 \mathrm{~Hz}), 6.87(\mathrm{dd}, 2 \mathrm{H}, J=2.6,7.1 \mathrm{~Hz}), 5.87-5.73(\mathrm{~m}, 1 \mathrm{H}), 5.19-5.11(\mathrm{~m}, 2 \mathrm{H})$, 4.67(t, $1 \mathrm{H}, J=6.3 \mathrm{~Hz}), 3.80(\mathrm{~s}, 3 \mathrm{H}), 2.52-2.48(\mathrm{t}, 2 \mathrm{H}, J=7.2 \mathrm{~Hz}), 2.00(\mathrm{~s}, 1 \mathrm{H}) ; \mathrm{IR}$ $(\mathrm{KBr}): v \quad 3407,3075,3002,2933,2836,1640,1612,1513,1247 \mathrm{~cm}^{-1}$.

1-(4-chlorophenyl)-3-buten-1-ol (3j) ${ }^{11}{ }^{1} \mathrm{H}$ NMR (300MHz, $\left.\mathrm{CDCl}_{3}\right): \delta$ 7.34-7.26(m, $4 \mathrm{H}), 5.83-5.74(\mathrm{~m}, 1 \mathrm{H}), 5.20-5.14(\mathrm{~m}, 2 \mathrm{H}), 4.70(\mathrm{q}, 1 \mathrm{H}, J=3.3,4.2 \mathrm{~Hz}), 2.51-2.44(\mathrm{~m}$ 2H), 2.06(d, 1H, $J=3.2 \mathrm{~Hz})$; IR (KBr, film): $v$ 3386, 3078, 2929, 1641, 1493, 1091, $1014 \mathrm{~cm}^{-1}$

1-(2,4-dichlorophenyl)-3-buten-1-ol (3k) ${ }^{10}$ m.p.59.5 ${ }^{\circ} \mathrm{C} ;{ }^{1} \mathrm{H} \quad \mathrm{NMR} \quad(300 \mathrm{MHz}$ $\left.\mathrm{CDCl}_{3}\right): \delta 7.5(\mathrm{~d}, 1 \mathrm{H}, J=8.7 \mathrm{~Hz}), 7.35(\mathrm{~d}, 1 \mathrm{H}, J=2.2 \mathrm{~Hz}), 7.26(\mathrm{~d}, 1 \mathrm{H}, J=5.3 \mathrm{~Hz})$ 5.86-5.80(m, 1H), 5.23-5.10(m, $3 \mathrm{H}), 2.64-2.59(\mathrm{~m}, 1 \mathrm{H}), 2.39-2.33(\mathrm{~m}, 1 \mathrm{H}), 2.16(\mathrm{~d}, 1 \mathrm{H}$ $J=3.3 \mathrm{~Hz})$; IR (KBr): $v \quad 3283,3080,2977,1639,1591,1471,1070 \mathrm{~cm}^{-1}$. 
1-cyclohexyl-3-buten-1-ol (3I) ${ }^{6}{ }^{1} \mathrm{H}$ NMR $\left(300 \mathrm{MHz}, \mathrm{CDCl}_{3}\right): \delta 5.91-5.77(\mathrm{~m}, 1 \mathrm{H})$, 5.16-5.11(m, 2H), 3.40-3.37(m, 1H), 2.38-2.30(m, 1H), 2.17-2.07(m, 1H), 1.88-0.99(m, 12H); IR (KBr, film): $v \quad 3391,3075,2925,2853,1640,1450 \mathrm{~cm}^{-1}$.

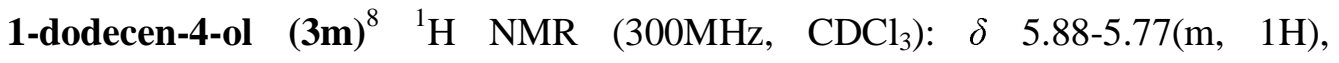
5.17-5.11(m, 2H), 3.65(d, $1 \mathrm{H}, J=3.4 \mathrm{~Hz}), 2.35-2.09(\mathrm{~m}, 2 \mathrm{H}), 1.59-1.27(\mathrm{~m}, 15 \mathrm{H})$, 0.86(t, 3H, $J=6.5 \mathrm{~Hz})$; IR (KBr, film): $v \quad 3361$, 3077, 2926, 2855, 1641, 1466, 912 $\mathrm{cm}^{-1}$.

1-nonen-4-ol (3n) ${ }^{12}{ }^{1} \mathrm{H}$ NMR $\left(300 \mathrm{MHz}, \mathrm{CDCl}_{3}\right): \delta 5.90-5.78(\mathrm{~m}, 1 \mathrm{H}), 5.17-5.11(\mathrm{~m}$, $2 \mathrm{H}), 3.63(\mathrm{~d}, 1 \mathrm{H}, J=3.7 \mathrm{~Hz}), 2.33-2.11(\mathrm{~m}, 2 \mathrm{H}), 1.62-1.25(\mathrm{~m}, 8 \mathrm{H}), 0.87(\mathrm{t}, 3 \mathrm{H}, J=6.7$ $\mathrm{Hz}$ ); IR (KBr, film): $v$ 3365, 3077, 2930, 2859, 1641, 1467, $1028 \mathrm{~cm}^{-1}$.

1-phenylhexa-1, 5-dien-3-ol (3o) ${ }^{8}{ }^{1} \mathrm{H}$ NMR (300MHz, $\left.\mathrm{CDCl}_{3}\right): \delta 7.40-7.22(\mathrm{~m}, 5 \mathrm{H})$, 6.59(d, 1H, $J=15.8 \mathrm{~Hz}), 6.29-6.21(\mathrm{~m}, 1 \mathrm{H}), 5.91-5.82(\mathrm{~m}, 1 \mathrm{H}), 5.22-5.15(\mathrm{~m}, 2 \mathrm{H})$, 4.39-4.35(m, 1H), 2.45-2.38(m, 2H), 1.78(d, 1H, J=5.0 Hz); IR (KBr, film): $v \quad 3366$, $3026,2978,2928,1641,1599,1494,1030 \mathrm{~cm}^{-1}$.

2-hydroxypent-4-enoic acid $\quad(\mathbf{3 p})^{13}{ }^{1} \mathrm{H} \quad \mathrm{NMR} \quad\left(300 \mathrm{MHz}, \quad \mathrm{CD}_{3} \mathrm{COCD}_{3}\right): \quad \delta$ 5.96-5.83(m, 1H), 5.16-5.03(st, 2H, $J=9.5 \mathrm{~Hz}), 4.21(\mathrm{~s}, 1 \mathrm{H}), 2.56-2.39(\mathrm{~m}, 2 \mathrm{H})$; IR (KBr, film): $v$ 3398, 3080, 2983, 2941, 1727, 1643, 1435, 1398, 1215, 1085, $919 \mathrm{~cm}^{-1}$ Boc- $D$-phenylalaninal (4) (synthesized according to the literature ${ }^{3}$ ): $[\alpha]_{D}^{27}=+32.5$ $\left(c=1.35\right.$ in $\left.\mathrm{CH}_{3} \mathrm{OH}\right)\left(\right.$ literature ${ }^{14}:$ Boc- $\boldsymbol{L}$-phenylalaninal, $[\alpha]^{22}{ }_{\mathrm{D}}=-31, c=1.0$ in $\left.\mathrm{CH}_{3} \mathrm{OH}\right) ;{ }^{1} \mathrm{H} \mathrm{NMR}\left(300 \mathrm{MHz}, \mathrm{CDCl}_{3}\right): \delta=9.63(\mathrm{~s}, 1 \mathrm{H}), 7.34-7.16(\mathrm{~m}, 5 \mathrm{H}), 5.07(\mathrm{~s}, 1 \mathrm{H})$, 4.40(q, 1H, $J=6.3,6.9,5.9 \mathrm{~Hz}), 3.11(\mathrm{~d}, 2 \mathrm{H}, J=6.6 \mathrm{~Hz}), 1.43(\mathrm{~s}, 9 \mathrm{H}) ; \mathrm{IR}(\mathrm{KBr}): v$ $3369,3030,2980,2846,1732,1690,1520 \mathrm{~cm}^{-1}$. 
$(2 R, 3 R)$-(tert-butoxycarbonylamino)-1-phenyl-5-hexen-3-ol $(5 a)^{15}[\alpha]^{27}{ }_{D}=$ $+12.30\left(c=0.91\right.$ in $\left.\mathrm{CHCl}_{3}\right)$, (literature ${ }^{15}:(2 S, 3 S)-(t$-butoxycarbonylamino $)-1-$ phenyl-5-hexen-3-ol, $[\alpha]^{24}=-16.8, \mathrm{c}=1.0$ in $\left.\mathrm{CHCl}_{3}\right) ;{ }^{1} \mathrm{H} \mathrm{NMR}\left(300 \mathrm{MHz}, \mathrm{CDCl}_{3}\right): \delta$ 7.32-7.19(m, 5H), 5.90-5.81(m, 1H), 5.15(sd, 2H, $J=10.2,6.1 \mathrm{~Hz}), 4.60(\mathrm{~d}, 1 \mathrm{H}, J=$ $8.10 \mathrm{~Hz}), 3.80(\mathrm{~d}, 1 \mathrm{H}, J=4.5 \mathrm{~Hz}), 3.71(\mathrm{~d}, 1 \mathrm{H}, J=2.0 \mathrm{~Hz}), 2.97-2.69(\mathrm{~m}, 3 \mathrm{H})$, 2.35-2.23(m, 2H), 1.35(s, 1H); IR (KBr): $v$ 3360, 2980, 2930, 1685, 1644, $1528 \mathrm{~cm}^{-1}$. $(2 R, 3 S)$-(tert-butoxycarbonylamino)-1-phenyl-5-hexen-3-ol $(5 \mathbf{b})^{15}[\alpha]^{27}{ }_{\mathrm{D}}=$ $+18.97\left(c=1.03\right.$ in $\left.\mathrm{CHCl}_{3}\right),\left(\right.$ literature ${ }^{15}:(\mathbf{2 S}, \mathbf{3 R})$-(t-butoxycarbonylamino)-1phenyl-5-hexen-3-ol, $[\alpha]^{24}=-22.3, c=1.0$ in $\left.\mathrm{CHCl}_{3}\right) ;{ }^{1} \mathrm{H} \mathrm{NMR}\left(300 \mathrm{MHz}, \mathrm{CDCl}_{3}\right): \delta$ 7.32-7.18(m, 5H), 5.80-5.71(m, 1H), 5.10(d, 2H, $J=14.4 \mathrm{~Hz}), 4.87(\mathrm{~d}, 1 \mathrm{H}, J=8.7 \mathrm{~Hz})$, 3.73(q, $1 \mathrm{H}, J=8.6,7.9,7.5 \mathrm{~Hz}), 3.62-3.59(\mathrm{~m}, 1 \mathrm{H}), 2.92-2.83(\mathrm{~m}, 2 \mathrm{H}), 2.26-2.22(\mathrm{~m}$, 2H), 2.16(s, 1H), 1.41(s, 9H); IR (KBr): $v$ 3425, 3376, 3026, 2981, 2935, 1687, 1670, $1644,1526,1172 \mathrm{~cm}^{-1}$

1-phenyl-3-penten-1-ol $\quad(\mathbf{6 a})(Z$-isomer $){ }^{16} \quad{ }^{1} \mathrm{H} \quad \mathrm{NMR} \quad\left(300 \mathrm{MHz}, \quad \mathrm{CDCl}_{3}\right): \quad \delta$ 7.40-7.27(m, 5H), 5.68-5.62(m, 1H), 5.47-5.41(M, 1H), 4.75-4.69(m, $1 \mathrm{H})$, $2.63-2.45(\mathrm{~m}, 2 \mathrm{H}), 2.02(\mathrm{~d}, 1 \mathrm{H}, J=3.0 \mathrm{~Hz}), 1.62-1.60(\mathrm{dt}, 3 \mathrm{H}, J=0.8,7.7 \mathrm{~Hz}) ; \mathrm{IR}(\mathrm{KBr}$ film): $v 3378,3025,2916,1493,1453,1048 \mathrm{~cm}^{-1}$.

2-methyl-1-phenylbut-3-en-1-ol $\quad(7 \mathbf{a})^{17}$ syn/anti $=$ 58/42. ${ }^{1} \mathrm{H}$ NMR $(300 \mathrm{MHz}$, $\left.\mathrm{CDCl}_{3}\right): \delta 7.29-7.19(\mathrm{~m}, 5 \mathrm{H}), 5.80-5.63(\mathrm{~m}, 1 \mathrm{H}), 5.17-5.10(\mathrm{~m}, 2 \times 0.42 \mathrm{H})($ anti)$)$, 5.01-4.96(m, 2 x $0.58 \mathrm{H})(s y n), 4.53(\mathrm{dd}, 1$ x $0.58 \mathrm{H}, J=3.8,4.5 \mathrm{~Hz})(\operatorname{syn}), 4.27(\mathrm{dd}, 1 \mathrm{x}$ $0.42 \mathrm{H}, J=2.1,5.8)($ anti) $), 2.55-2.48(\mathrm{~m}, 1 \times 0.58 \mathrm{H})(\mathrm{syn}), 2.42-2.37(\mathrm{~m}, 1 \times 0.42$ $\mathrm{H})($ anti), 2.1(d, 1 x $0.42 \mathrm{H}, J=2.6)($ anti $), 1.89(\mathrm{~d}, 1 \times 0.58 \mathrm{H}, J=3.2 \mathrm{~Hz})(\mathrm{syn}), 0.93(\mathrm{~d}$, 
3x $0.58 \mathrm{H}, J=7.0 \mathrm{~Hz})(s y n), 0.78(\mathrm{~d}, 3 \times 0.42 \mathrm{H}, J=6.8 \mathrm{~Hz})($ anti). $)$; IR (KBr, film): $v$ $3422,3065,2973,2874,1638,1493,1453,1017,913,701 \mathrm{~cm}^{-1}$.

1-(4-nitophenyl)-3-penten-1-ol (6b) $\left(Z\right.$-isomer) ${ }^{16}{ }^{1} \mathrm{H}$ NMR $\left(300 \mathrm{MHz}, \mathrm{CDCl}_{3}\right): \delta$ 8.18(d, $2 \mathrm{H}, J=8.8 \mathrm{~Hz}), 7.53(\mathrm{~d}, 2 \mathrm{H}, J=8.4 \mathrm{~Hz}), 5.73-5.67(\mathrm{~m}, 1 \mathrm{H}), 5.38(\mathrm{dddd}, 1 \mathrm{H}, J=$ $1.2,2.1,2.7,3.8 \mathrm{~Hz}), 4.82(\mathrm{t}, 1 \mathrm{H}, J=6.4 \mathrm{~Hz}), 2.55-2.48(\mathrm{~m}, 2 \mathrm{H}), 2.30(\mathrm{~s}, 1 \mathrm{H}), 1.58(\mathrm{~d}$, 3H, $J=7.2 \mathrm{~Hz}$ ); IR (KBr, film): $v$ 3420, 3079, 3020, 2919, 2856, 1604, 1519, 1347 , $1055,855,701 \mathrm{~cm}^{-1}$.

2-methyl-1-(4-nitrophenyl)-3-buten-1-ol $\quad(\mathbf{7 b})^{18} \quad$ syn/anti $=55 / 45 . \quad{ }^{1} \mathrm{H} \quad \mathrm{NMR}$ $\left(300 \mathrm{MHz}, \mathrm{CDCl}_{3}\right): \delta 8.22-8.19(\mathrm{~d}, 2 \mathrm{H}, J=8.7 \mathrm{~Hz}), 7.56-7.47(\mathrm{~m}, 2 \mathrm{H}), 5.78-5.72(\mathrm{~m}$, 1H), 5.23-5.06(m, 2H), 4.78(s, $1 \times 0.55 \mathrm{H})(s y n), 4.51(\mathrm{~d}, 1 \mathrm{x} 0.45 \mathrm{H},, J=6.6 \mathrm{~Hz})($ anti), 2.61-2.48(m, 1H), 2.20(s, 1x 0.45H)(anti), 2.11(d, 1x 0.55H, $J=1.09 \mathrm{~Hz})($ syn), 0.96(d, 3x 0.55H, $J=6.7 \mathrm{~Hz})(s y n), 0.92(\mathrm{~d}, 3 \times 0.45 \mathrm{H},, J=7.0 \mathrm{~Hz})(a n t i) ; \mathrm{IR}(\mathrm{KBr}$, film): $v$ 3344, 3079, 2966, 1713, 1640, 1605, 1519, 1347, 1013, 857, $702 \mathrm{~cm}^{-1}$.

1-(3-methoxyphenyl)-3-penten-1-ol (6c) (Z-isomer) ${ }^{16}{ }^{1} \mathrm{H} \mathrm{NMR}\left(300 \mathrm{MHz}, \mathrm{CDCl}_{3}\right)$ : $\delta \quad 7.28-7.23(\mathrm{~m}, \quad 1 \mathrm{H}), \quad 6.95-6.93(\mathrm{~m}, \quad 2 \mathrm{H}), \quad 6.83-6.79(\mathrm{~m}, \quad 1 \mathrm{H}), \quad 5.67-5.61(\mathrm{~m}, \quad 1 \mathrm{H})$, 5.47-5.40(m, 1H), 4.71-4.66(m, 1H), 3.81(s, 3H), 2.59-2.43(m, 2H), 2.09(d, 1H, $J=$ $2.8 \mathrm{~Hz}), 1.60(\mathrm{~d}, 3 \mathrm{H}, J=7.5 \mathrm{~Hz})$; IR (KBr, film): $v$ 3421, 2939, 2836, 1655, 1602, $1586,1488,1260,1044,874,701 \mathrm{~cm}^{-1}$.

2-methyl-1-(3-methoxyphenyl)-3-buten-1-ol $(\mathbf{7 c})^{19}$ syn/anti $=60 / 40 .{ }^{1} \mathrm{H}$ NMR $\left(300 \mathrm{MHz}, \mathrm{CDCl}_{3}\right): \delta \quad 7.28-7.21(\mathrm{~m}, 1 \mathrm{H}), \quad 6.94-6.79(\mathrm{~m}, \quad 3 \mathrm{H}), \quad 5.83-5.71(\mathrm{~m}, \quad 1 \mathrm{H})$, 5.22-5.16(m, 1x 0.4H)(anti), 5.08-5.03(m, 1x $0.6 \mathrm{H})($ syn $), 4.57(\mathrm{~d}, 1 \mathrm{x} 0.6 \mathrm{H}, J=4.3$ $\mathrm{Hz})($ syn), 4.31(d, 1x 0.4H, $J=7.5 \mathrm{~Hz})($ anti), 3.80(s, 3H), 2.58-2.46(m, 1H), 2.19(s, 1x 
$0.4 \mathrm{H})($ anti), 2.01(s, 1x 0.6H)(syn), 0.99(d, 3x 0.6Hz, $J=6.9 \mathrm{~Hz})($ syn $), 0.86(\mathrm{~d}, 3 \mathrm{x}$ $0.4 \mathrm{H}, J=7.0 \mathrm{~Hz})($ anti); IR (KBr, film): $v$ 3436, 3078, 2964, 2935, 2836, 1638, 1602 , $1586,1455,1260,1042,915,703 \mathrm{~cm}^{-1}$.

Dodec-2-en-5-ol (6d) ${ }^{20} \quad{ }^{1} \mathrm{H} \quad \mathrm{NMR} \quad\left(300 \mathrm{MHz}, \mathrm{CDCl}_{3}\right): \delta$ 5.69-5.62( $\left.\mathrm{m}, 1 \mathrm{H}\right)$, 5.49-5.42(m, 1H), 3.63(d, $1 \mathrm{H}, J=3.4 \mathrm{~Hz}), 2.20(\mathrm{t}, 2 \mathrm{H}, J=7.2,6.3 \mathrm{~Hz}), 1.66(\mathrm{~d}, 3 \mathrm{H}, J=$ $6.6 \mathrm{~Hz}), 1.63-1.29(\mathrm{~m}, 13 \mathrm{H}), 0.85(\mathrm{t}, 3 \mathrm{H}, J=7.0 \mathrm{H})$; IR (KBr, film): $v$ 3356, 3017, 2927 , $2856,1658,1464,1261,1022,805,705 \mathrm{~cm}^{-1}$.

The preparation of tributyltin ester of $\boldsymbol{p}$-nitrobenzoic acid (similar with the

literature $\left.{ }^{4}\right)$ : To a stirred solution of $p$-nitrobenzoic acid sodium salt $(1.13 \mathrm{~g}, 6 \mathrm{mmol})$ in water $(12 \mathrm{~mL})$ was added a solution of $\mathrm{Bu}_{3} \mathrm{SnCl}(1.67 \mathrm{~g}, 5 \mathrm{mmol})$ in petroleum ether (5 mL) at $0{ }^{\circ} \mathrm{C}$. After stirring for $10 \mathrm{~min}$, the mixture was extracted with $\mathrm{CH}_{2} \mathrm{Cl}_{2}(2 \mathrm{x}$ $10 \mathrm{~mL}$ ). The organic layer was dried over $\mathrm{Na}_{2} \mathrm{SO}_{4}$. After evaporation of the solvent under reduced pressure, the pure product was obtained. Yellow oil. 2.27g, yield of 99\%. ${ }^{1} \mathrm{H}$ NMR $\left(300 \mathrm{MHz}, \mathrm{CDCl}_{3}\right): \delta 8.27(\mathrm{dd}, 4 \mathrm{H}, J=7.0 \mathrm{~Hz}), 1.71-1.62(\mathrm{~m}, 6 \mathrm{H})$, 1.43-1.31(m, 12H), 0.95(t, 9H, $J=7.2 \mathrm{~Hz})$; IR (KBr, film): $v$ 3108, 3051, 2957, 2871, 1654, 1604, 1527, 1330, 1128, 1015, 831, $595 \mathrm{~cm}^{-1}$; MS (70 eV, EI): 400(M-57+1)+; Anal. Calcd for $\mathrm{C}_{19} \mathrm{H}_{31} \mathrm{NO}_{4} \mathrm{Sn}$ (456.16): C 50.03; H 6.85; N 3.07; Found: C 49.95; H $6.96 ;$ N 2.83.

\section{References}


1 Jones, W. J.; Davies, W. Z.; Bowden, S. T.; Edwards, C.; Daris, V. V.; Thomas, L. H. J. Chem. Soc. 1947, 1446-1450.

2 Hoffmann, R. W.; Feussner, G.; Zeiss, H-J.; Schulz, S. J. Organomet. Chem. 1980, $18,321-329$

3 Oishi, S.; Kamano, T.; Niida, A.; Odagaki, Y.; Hamanaka, N.;Yamamoto, M.; Ajito, K.; Tamamura, H.; Otaka, A.; Fujii, N. J. Org. Chem. 2002, 67, 6162-6273.

4 Max, F.; David, G.; Daniel, W.; Albert, Z. J. Organometal. Chem. 1967, 9, 83-88.

5 Aoyama, N.; Hamada, T.; Manabe, K.; Kobayashi, S. J. Org. Chem. 2003, 68, 7329-7333.

6 Fürstner, A.; Voigtländer, D. Synthesis 2000, 959-969.

7 Aggarwal, V. K.; Vennall, G. P. Synthesis 1998, 1822-1826.

8 Gomes, P.; Gosmini, C. Périchon, J. Synthesis 2003, 1909-1915.

9 Taeg-Su, J.; Gyochang, K.; Soon Bang, K.; Bong Yong, C.; Youseung, K. Synthesis 2003, 775-779.

10 Zhang, Q. S.; Liang, Zh. Y.; Curran, D. P. J. Org. Chem. 2000, 65, 8866- 8873.

11 Kobayashi, S.; Aoyama, N.; Manabe, K. Synlett 2002, 483-485.

12 Pilcher, A. S.; DeShong, P. J. Org. Chem. 1996, 61, 6901-6905.

13 Zannetti, M. T.; Walter, C.; Knorst, M.; Fessner, W-D.; Chem. Europ. J. 1999, 5, $1882-1890$.

14 Fehrentz, J.-A.; Pothion, C.; Califano, J.-C.; Loffet, A.t; Martinez, J. Tetrahedron Lett. 1994, 35, 9031-9034. 
15 Bonini, B. F.; Comes-Franchini, M.; Fochi, M.; Laboroi, F.; Mazzanti, G.; Ricci, A.; Varchi G. J. Org. Chem. 1999, 64, 8008-8013.

16 Nokami, J.; Yoshizane, K.; Matsuura, H.; Sumida, S. J. Am. Chem. Soc. 1998, 120, 6609-6610.

17 Tan, K-T.; Chng, S-S.; Cheng, H-S.; Loh, T-P. J. Am. Chem. Soc. 2003, 125 , $2958-2963$.

18 Batey, R.A.; Thadani, A. N.; Smil, D.V.; Lough, A. J. Synthesis 2000, 990 998.

19 Majee, A.; Das, A.R.; Ranu, B.C. Indian J. Chem. Sect. B, 1998, 37, 731 - 736.

20 Scala, A. D.; Garbacia, S.; Hélion, F.; Lannou, M-I.; Namy, J-L. Eur. J. Org. Chem. 2002, 2989-2995. 
1-phenyl-3-buten-1-ol (3a)

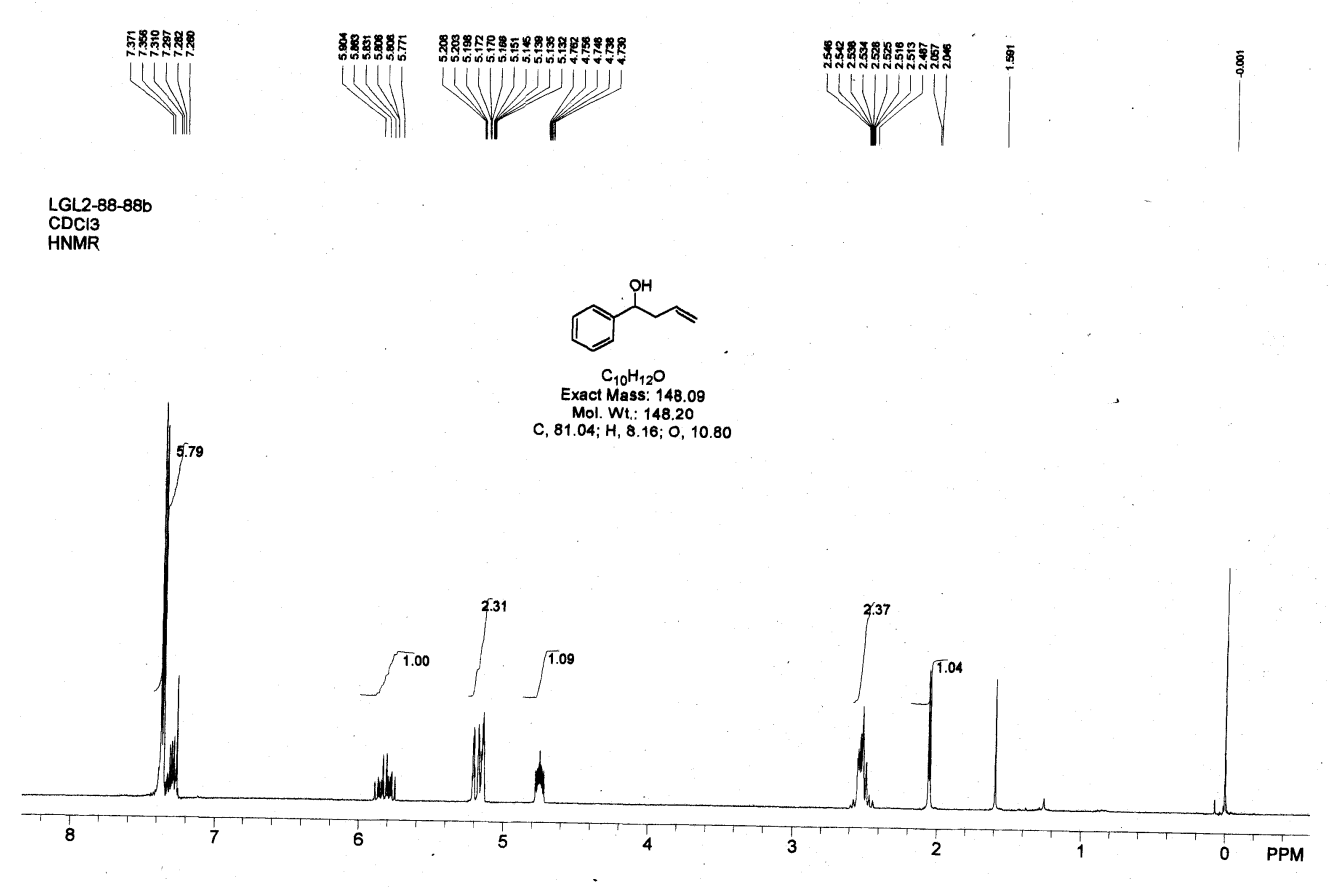

\section{1-(4-nitrophenyl)-3-buten-1-ol (3b)}




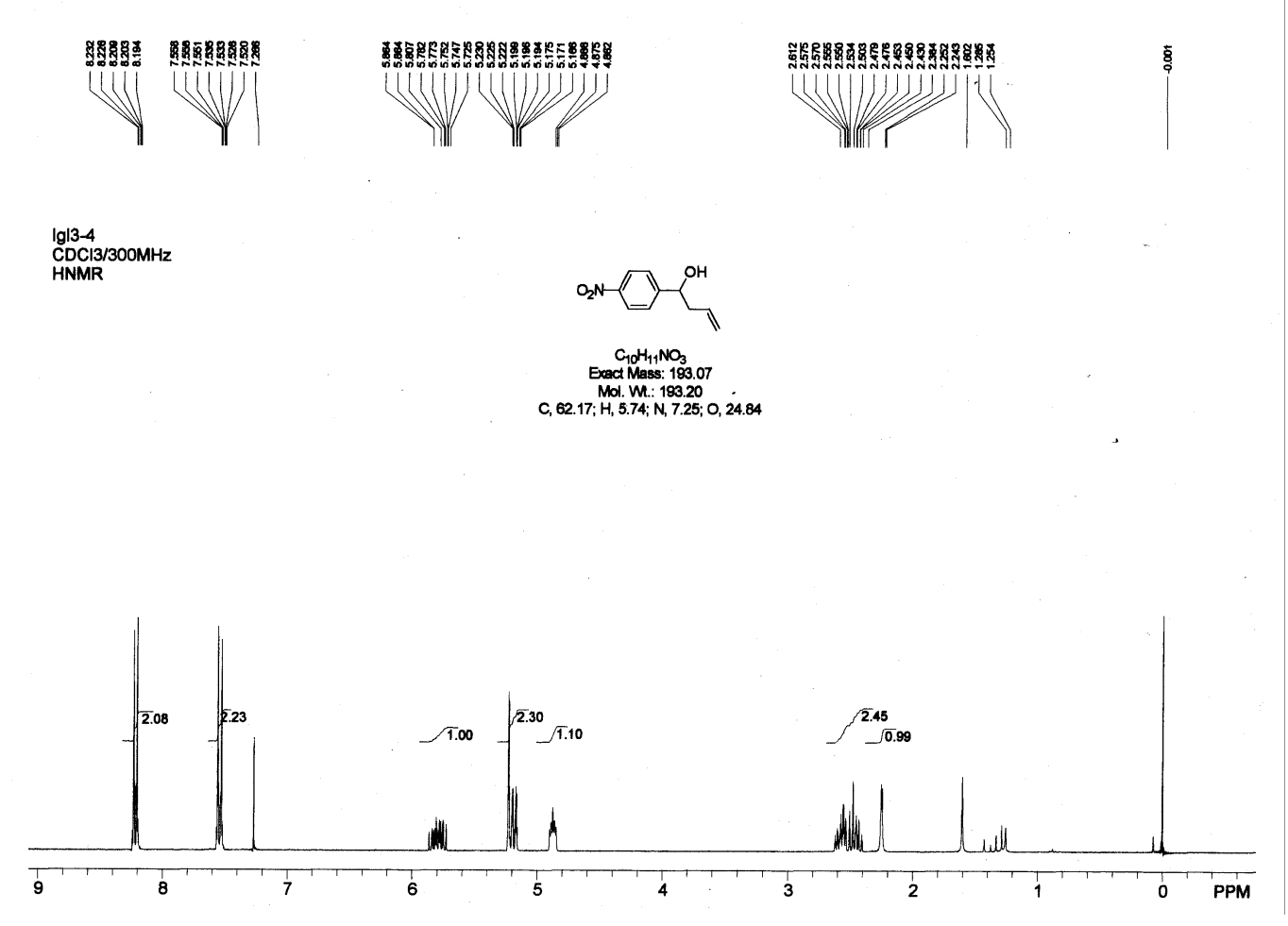

\section{1-(3-methoxyphenyl)-3-buten-1-ol}

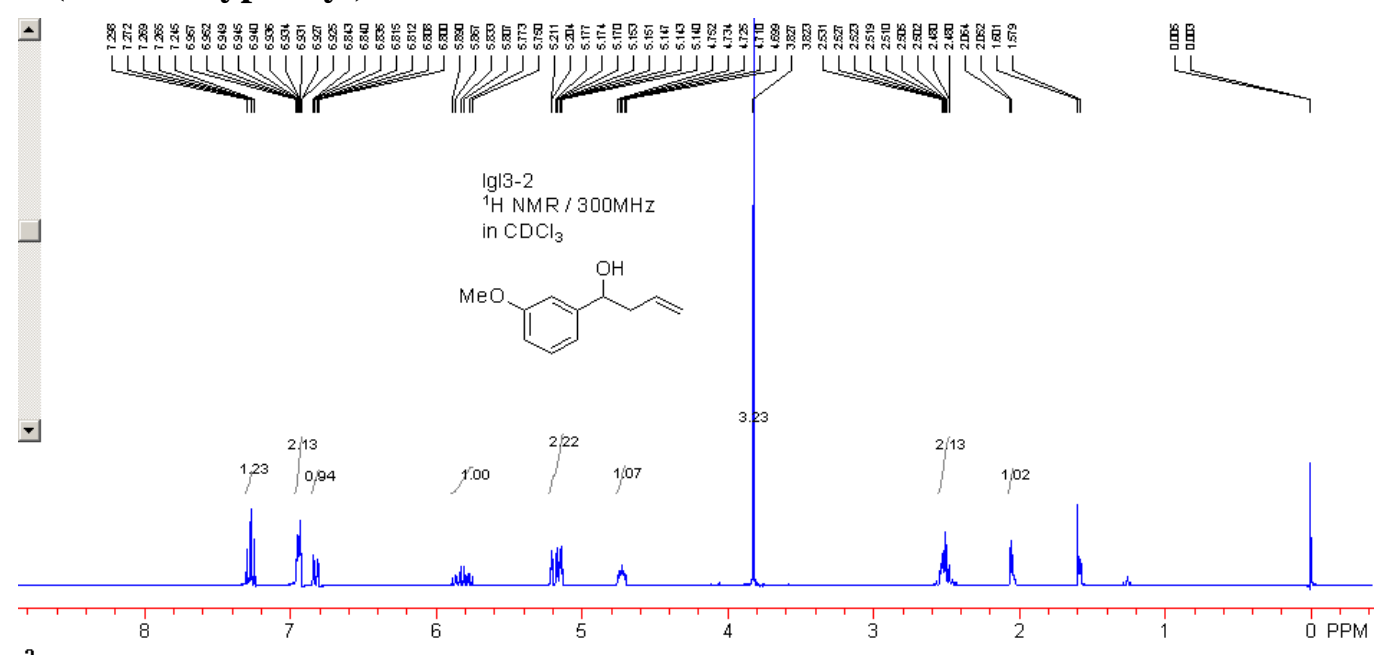

1-(4-n-butoxyphenyl)-3-buten-1-ol (3d) 


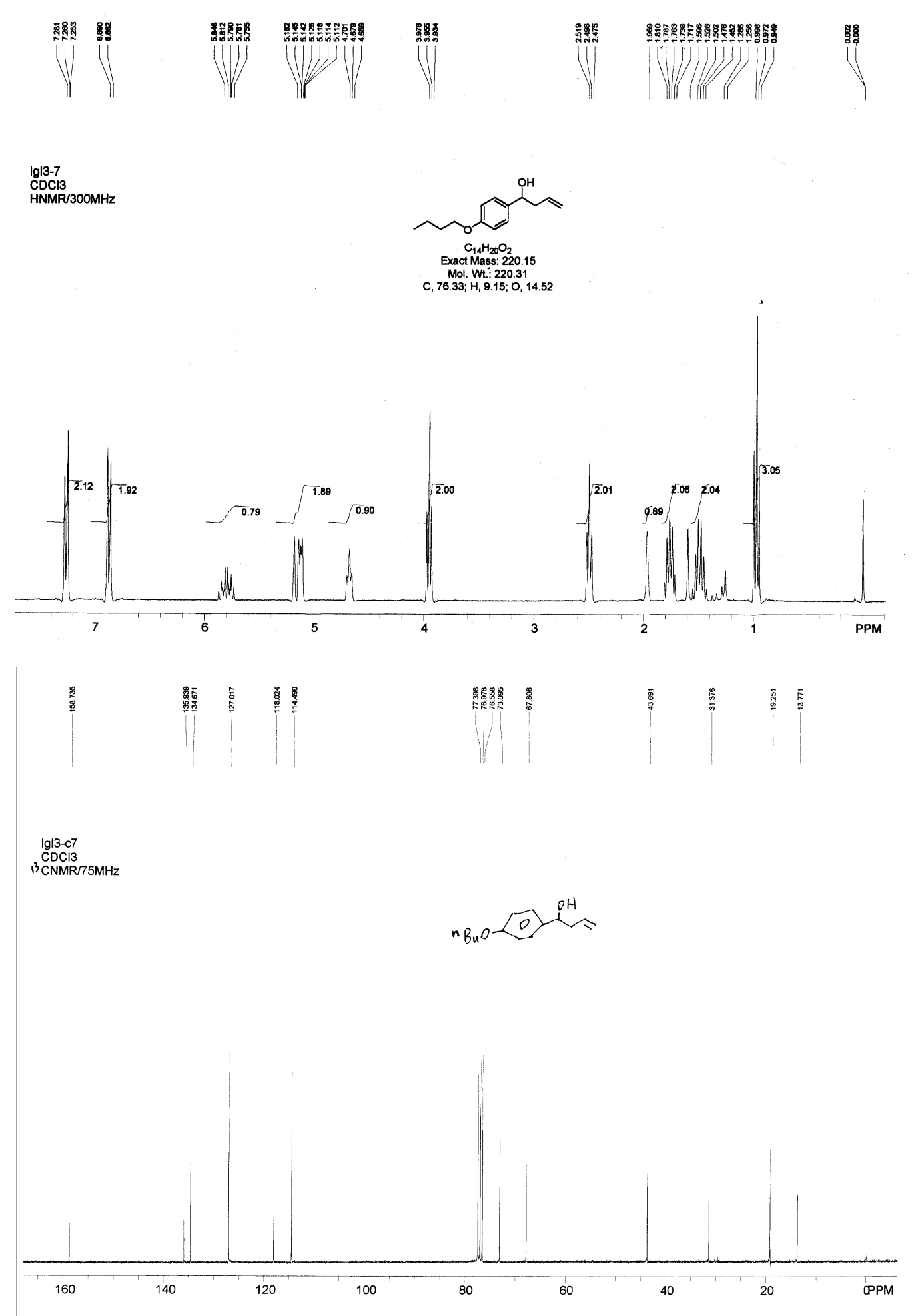

\section{1-(2-furyl)-3-buten-1-ol (3e)}




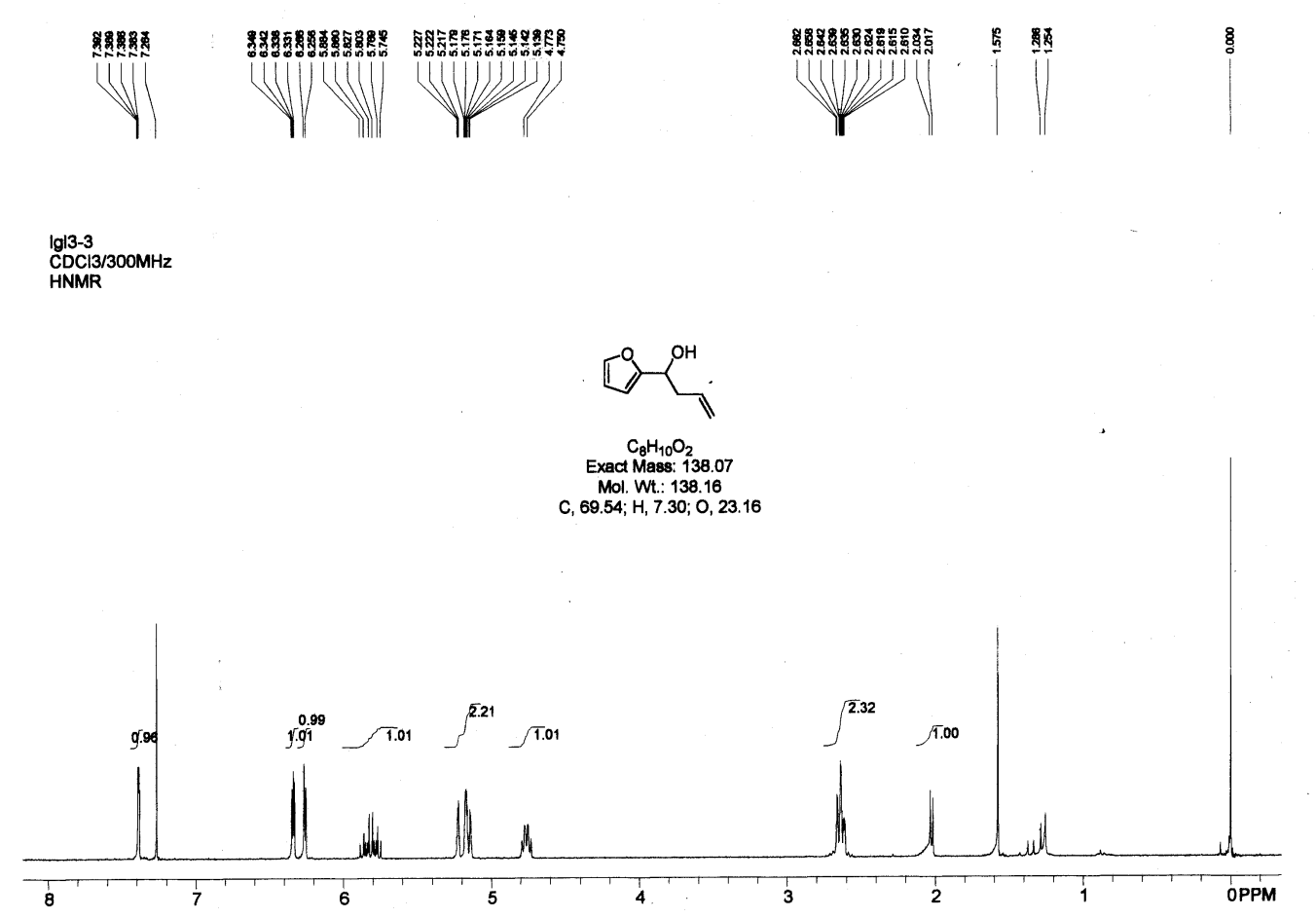

\section{1-(4-fluorophenyl)-3-buten-1-ol (3f)}

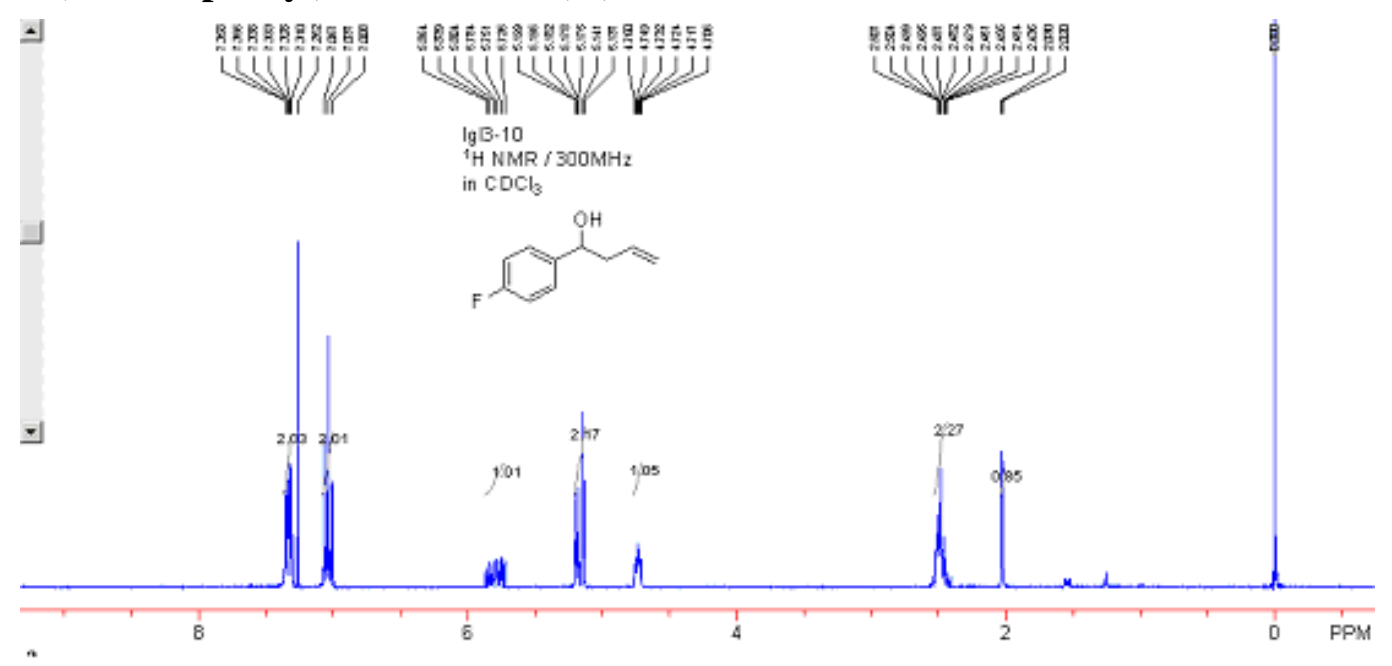

\section{1-(3,4,5-trifluorophenyl)-3-buten-1-ol}




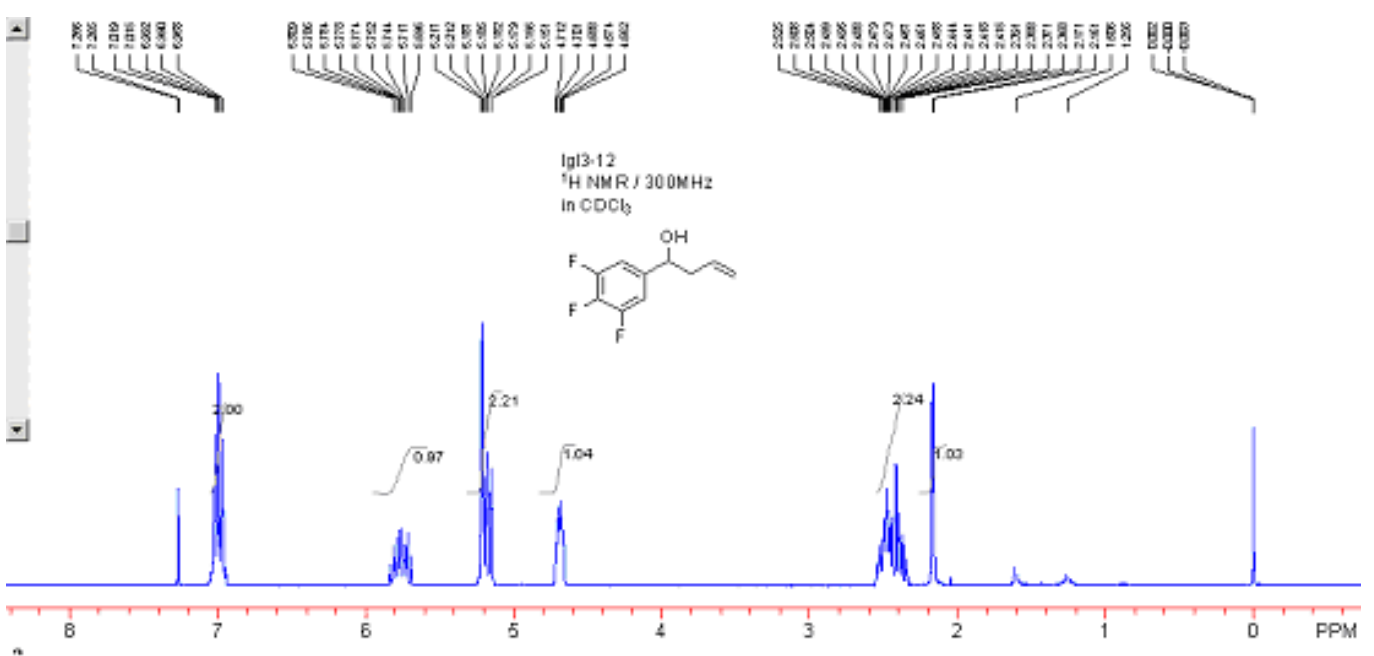

1-(4-acetamidophenyl)-3-buten-1-ol (3h)

VIV

$\lg 13.55$

HNMR3OOMHZ
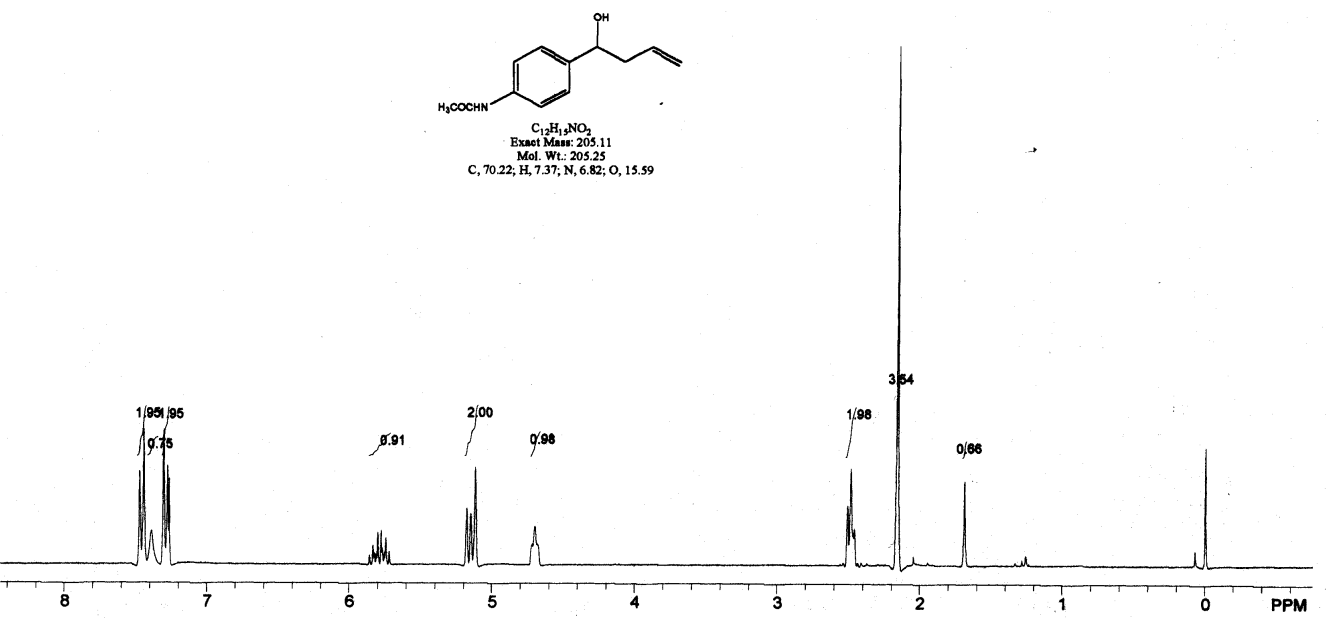

1-(4-methoxyphenyl)-3-buten-1-ol (3i) 


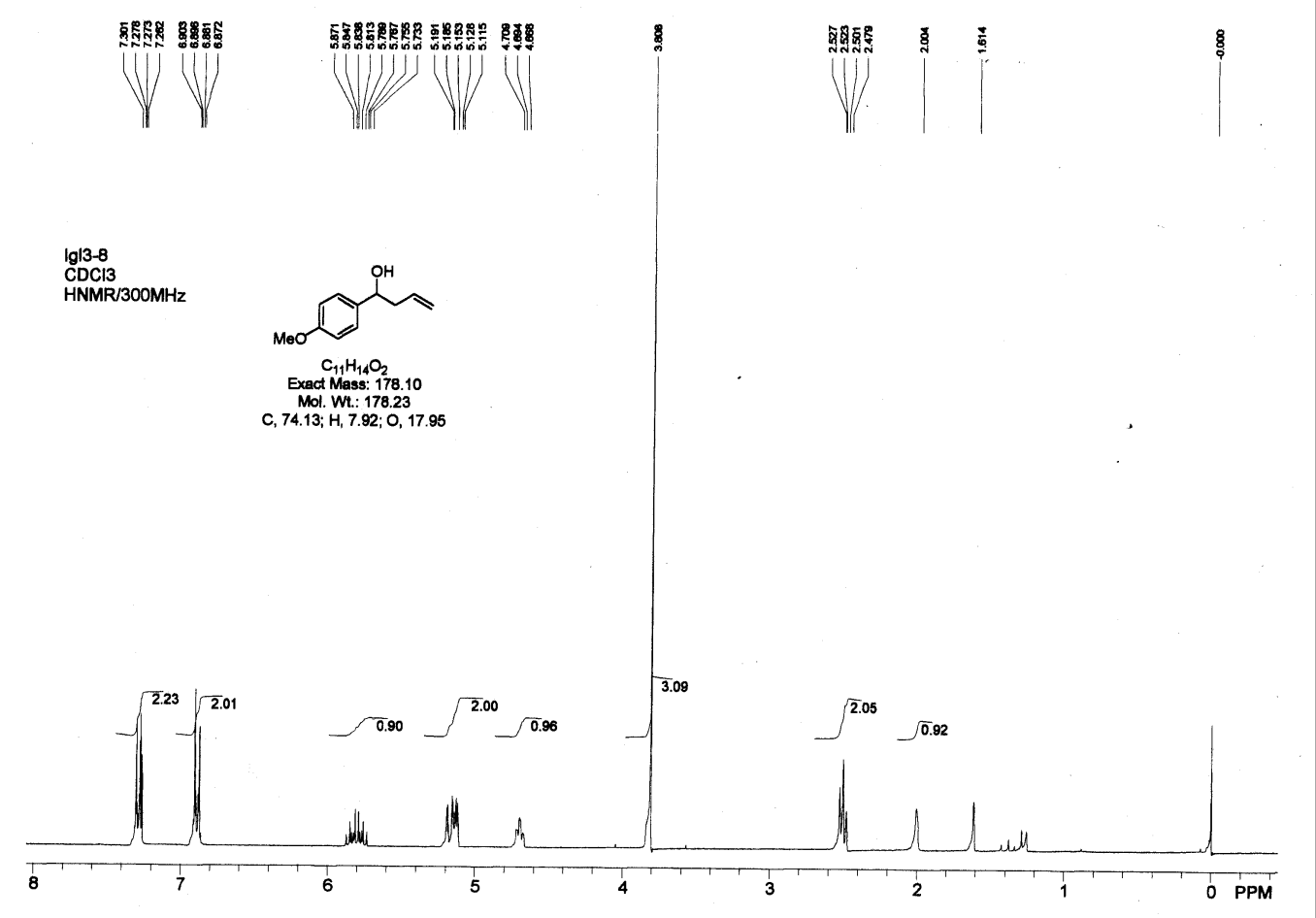

\section{1-(4-chlorophenyl)-3-buten-1-ol (3j)}
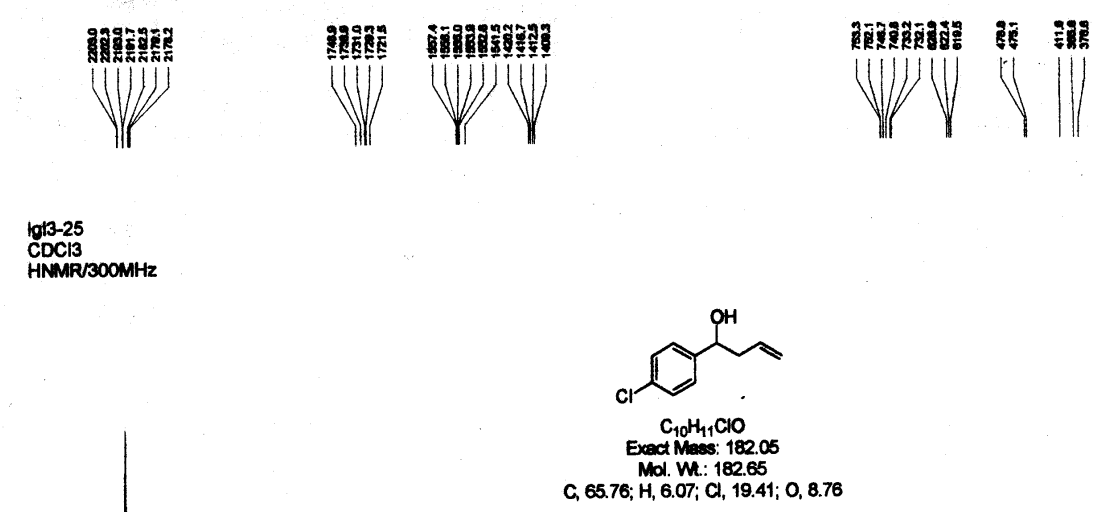


\section{1-(2,4-dichlorophenyl)-3-buten-1-ol}
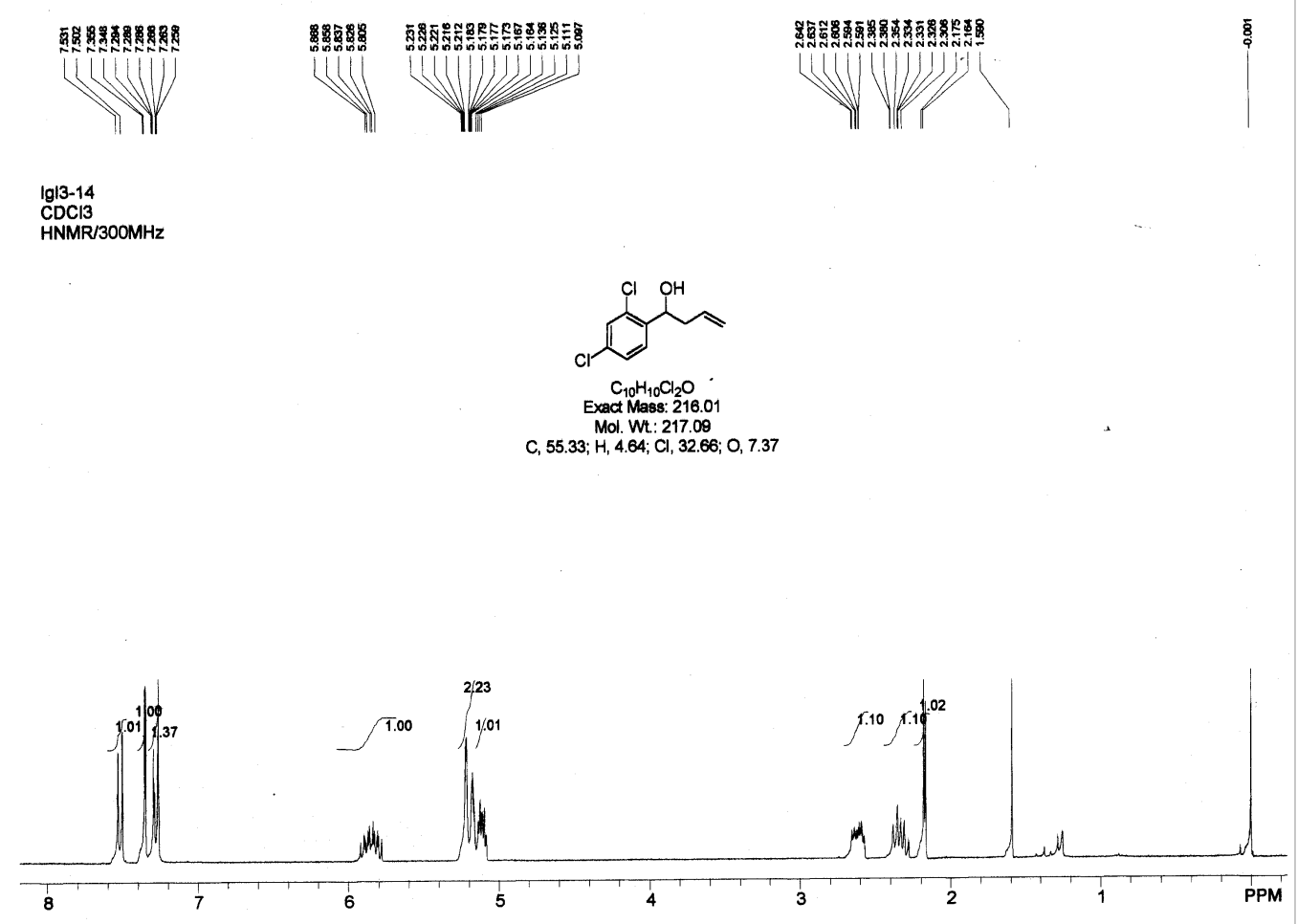

\section{1-cyclohexyl-3-buten-1-ol (3I)}
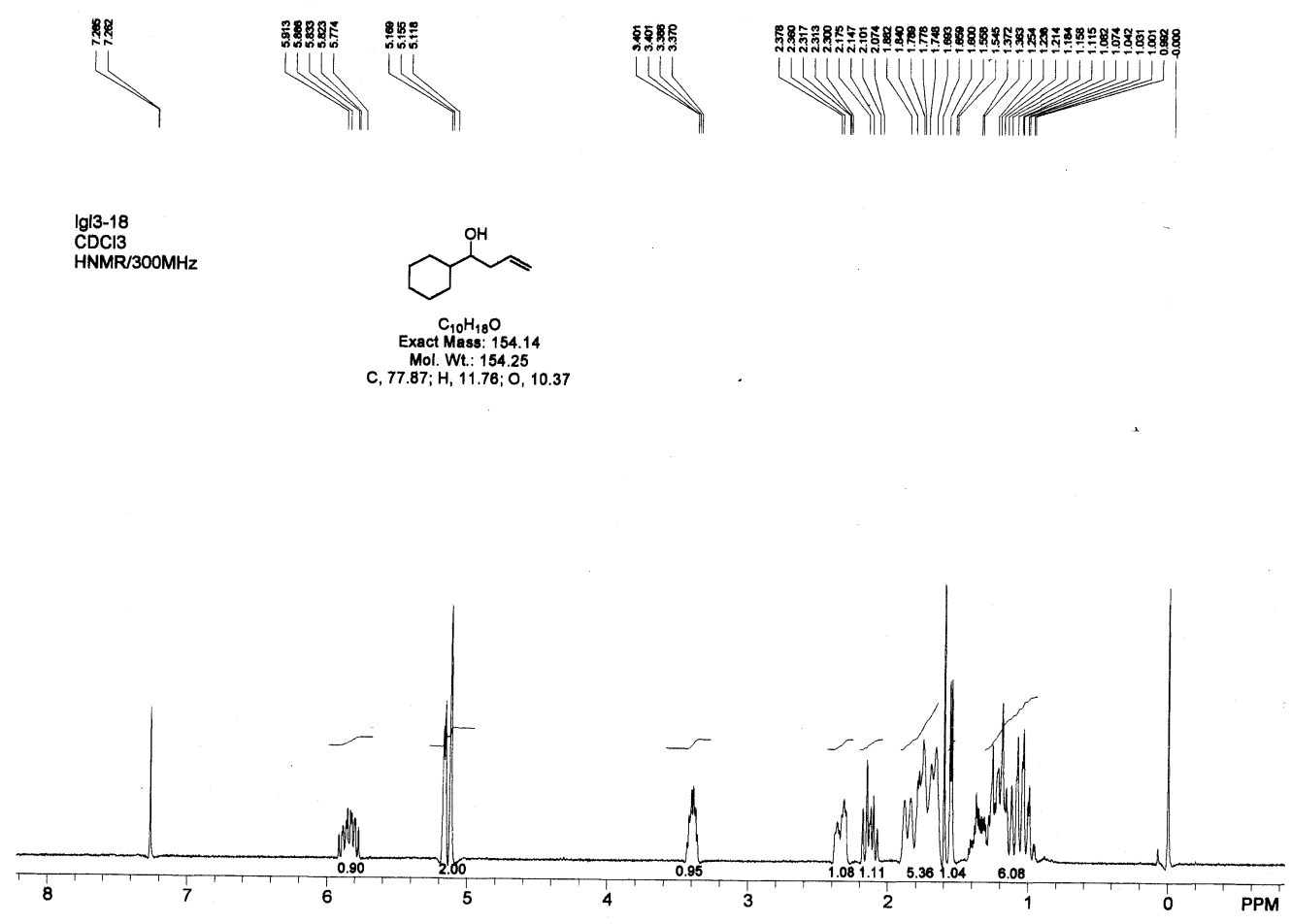


\section{1-dodecen-4-ol (3m)}
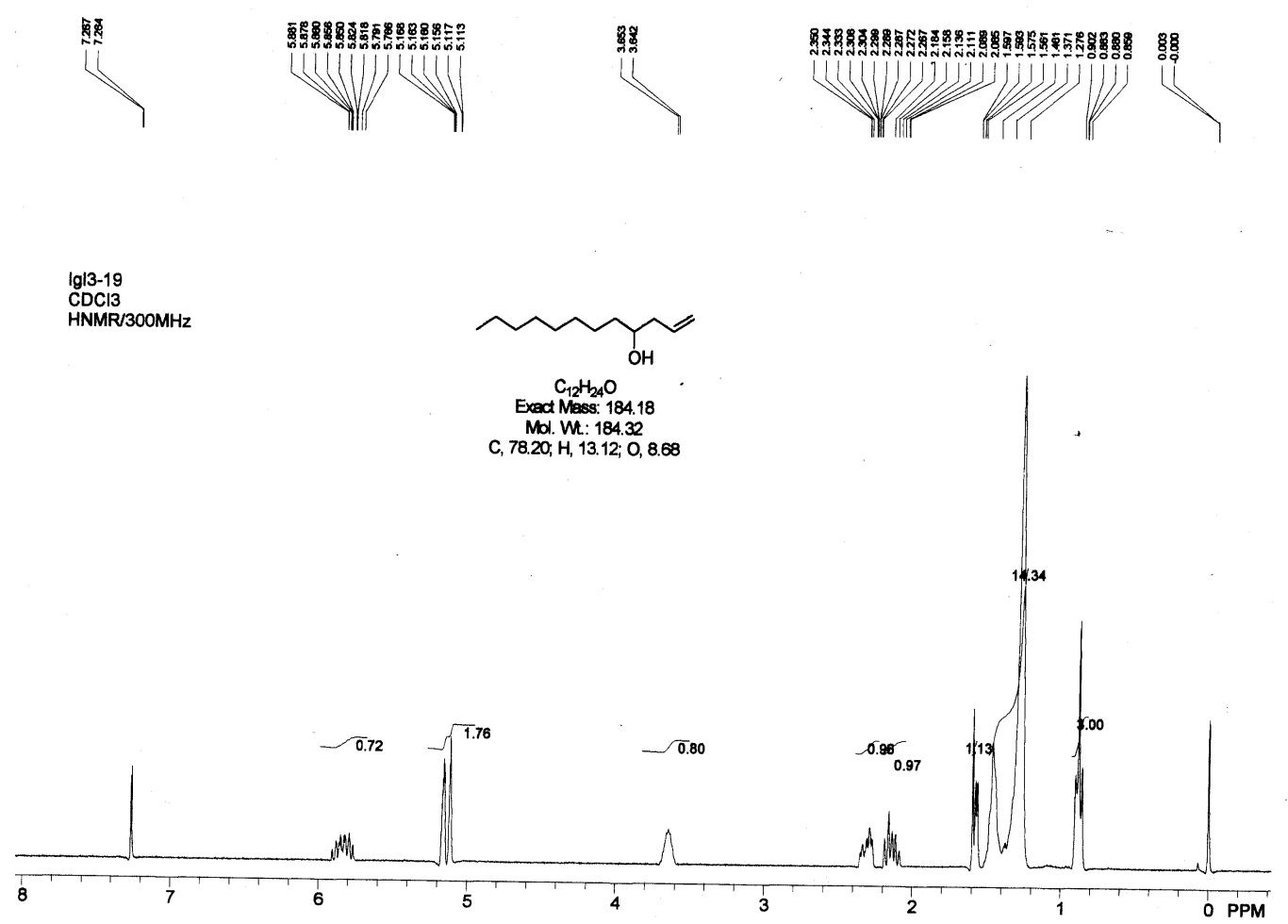

\section{1-nonen-4-ol (3n)}

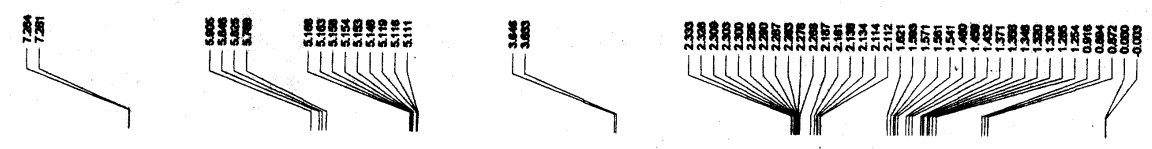

lols-27

HNMR/30OMHz

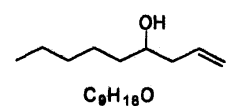

Exact Mass: 142.14

Mol. Wt: 142.24

C, $76.00 ; . H, 12.76 ; 0,11.25$

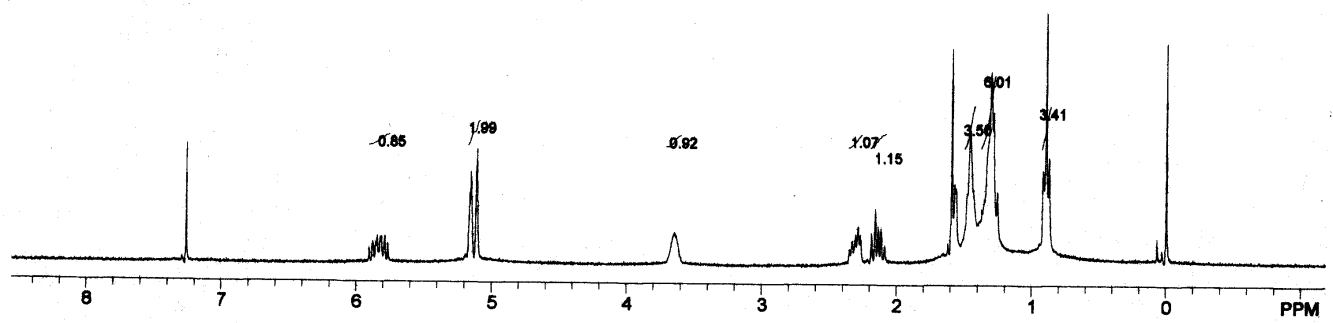




\section{1-phenylhexa-1, 5-dien-3-ol (3o)}
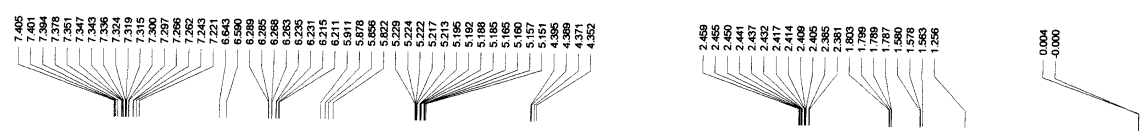

$\lg 14-4$

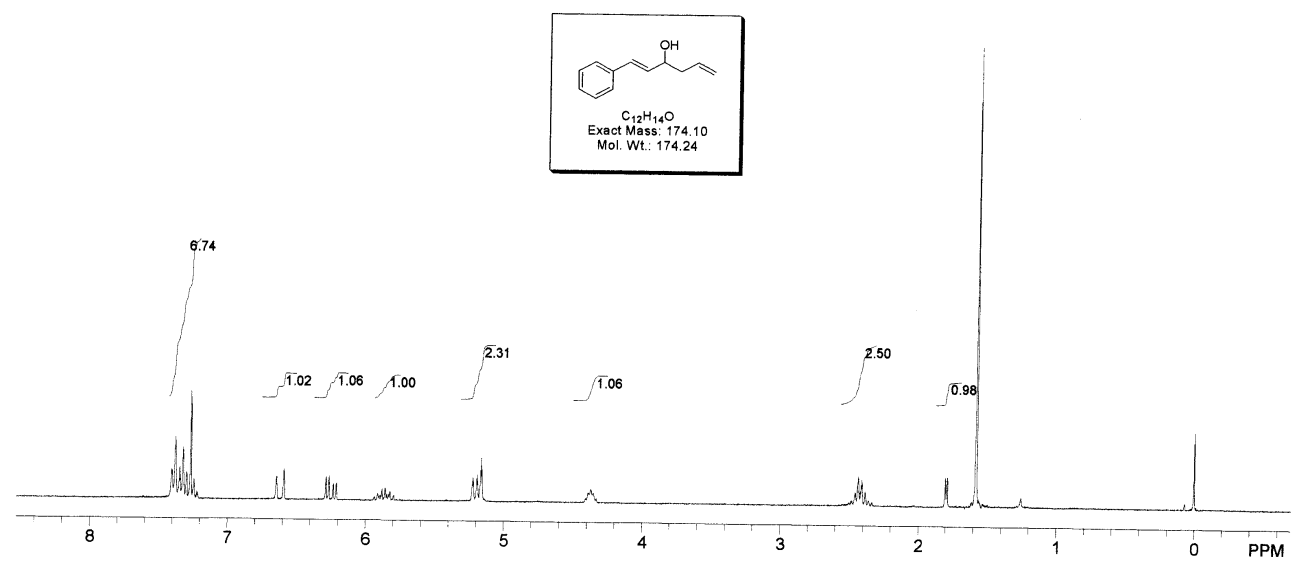

2-hydroxypent-4-enoic acid (3p)

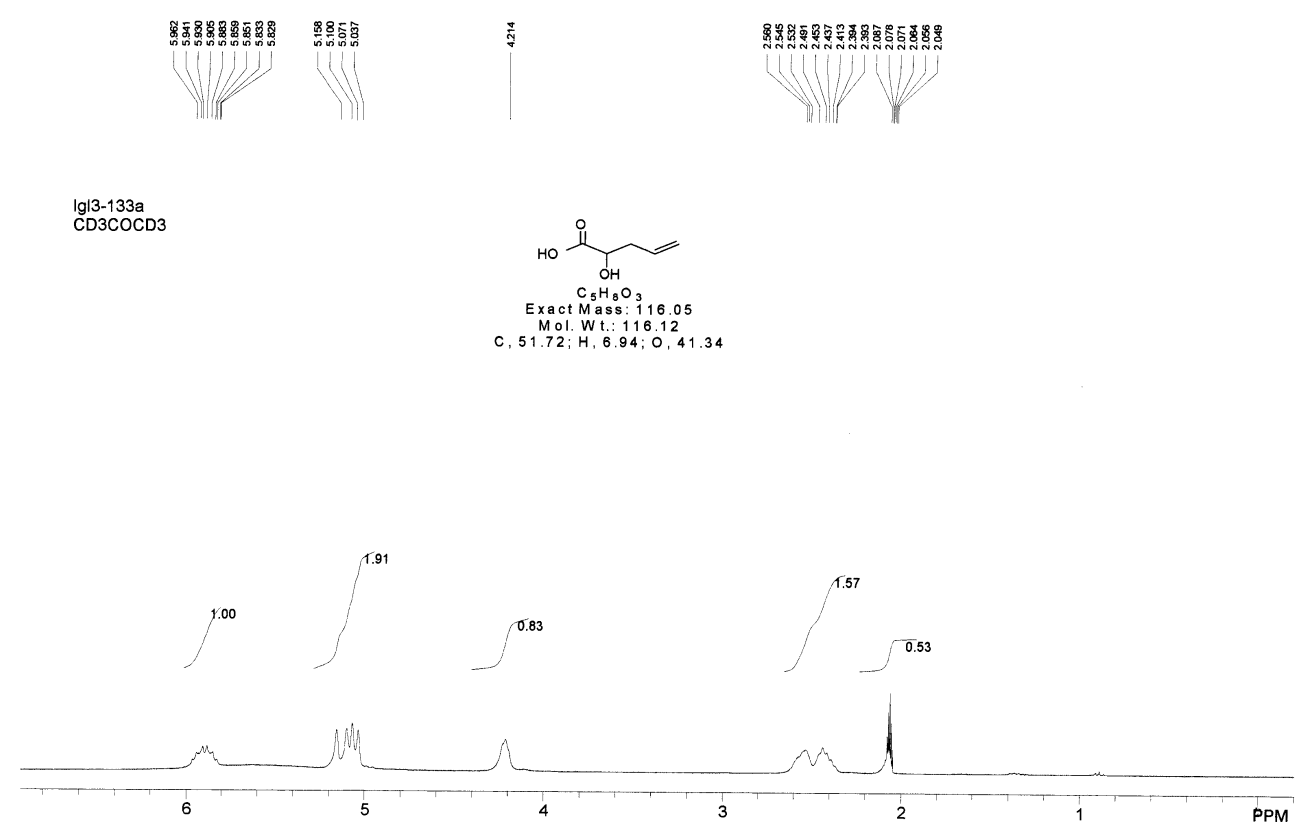

Boc- $D$-phenylalaninal (4) 


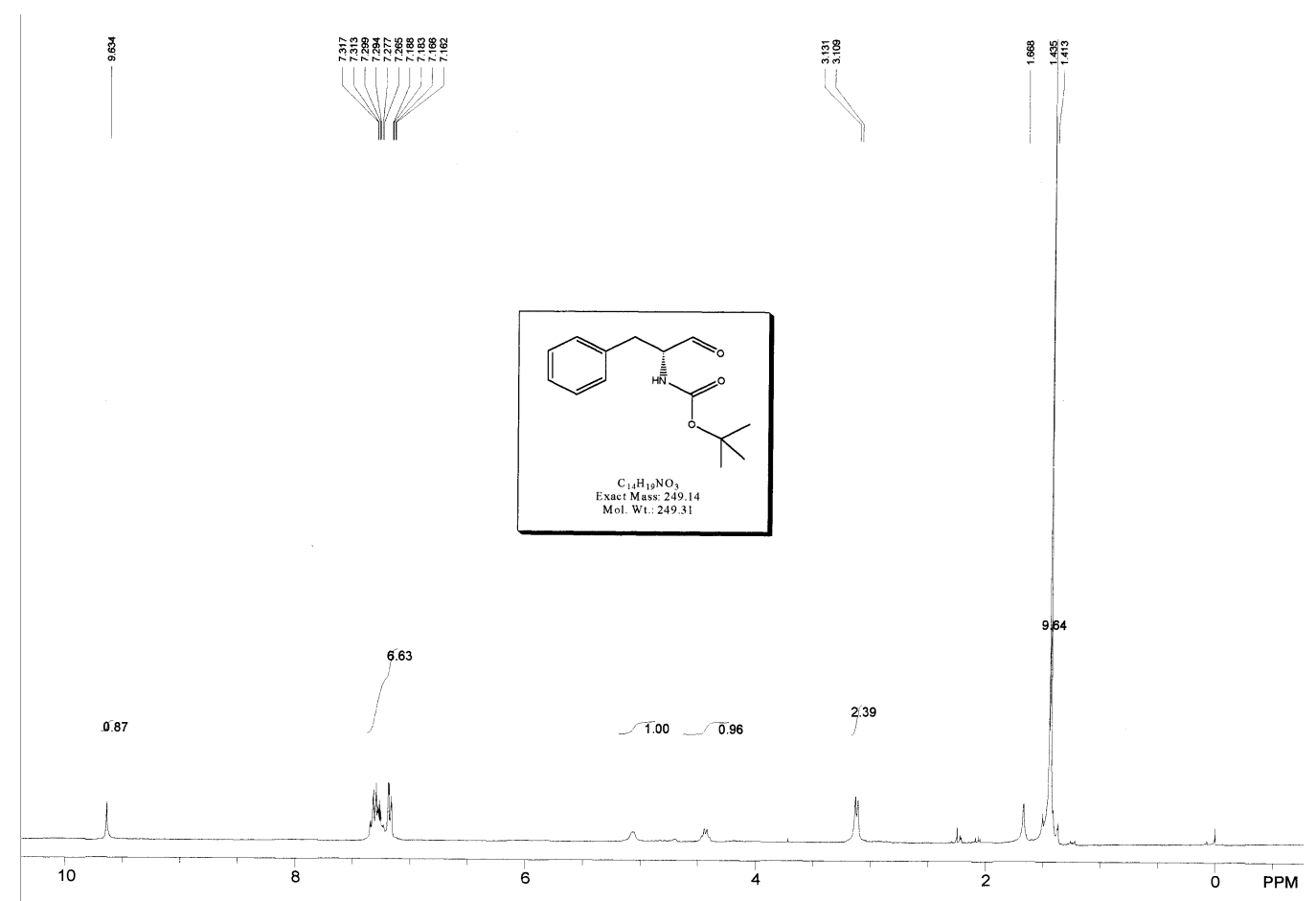

(2R, 3R)-(tert-butoxycarbonylamino)-1-phenyl-5-hexen-3-ol (5a)

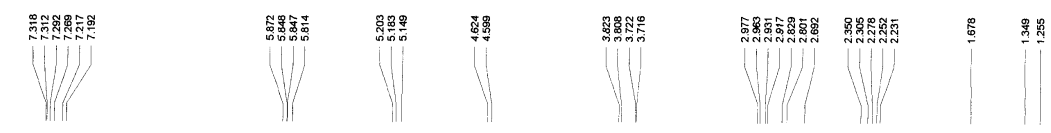

$$
\begin{aligned}
& \lg 14-22 \mathrm{C} \\
& \mathrm{CDCl}
\end{aligned}
$$

HNMR/300MHz
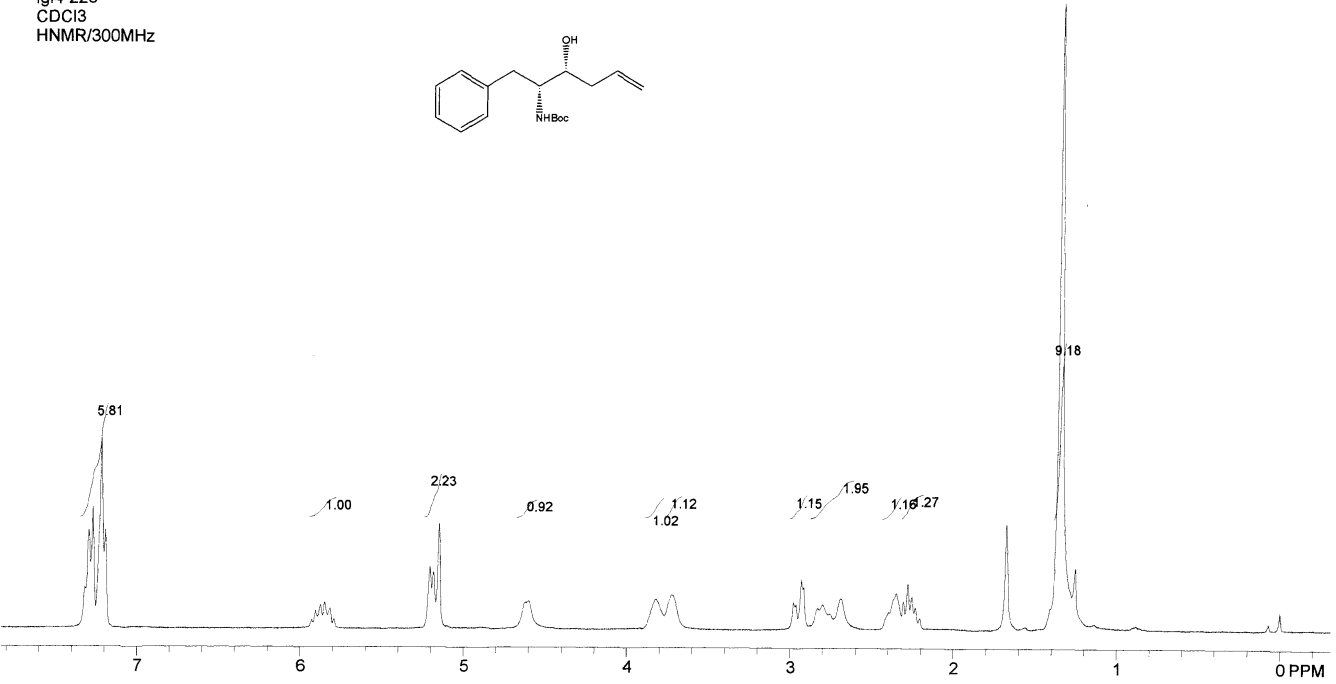

(2R, 3S)-(tert-butoxycarbonylamino)-1-phenyl-5-hexen-3-ol (5b) 


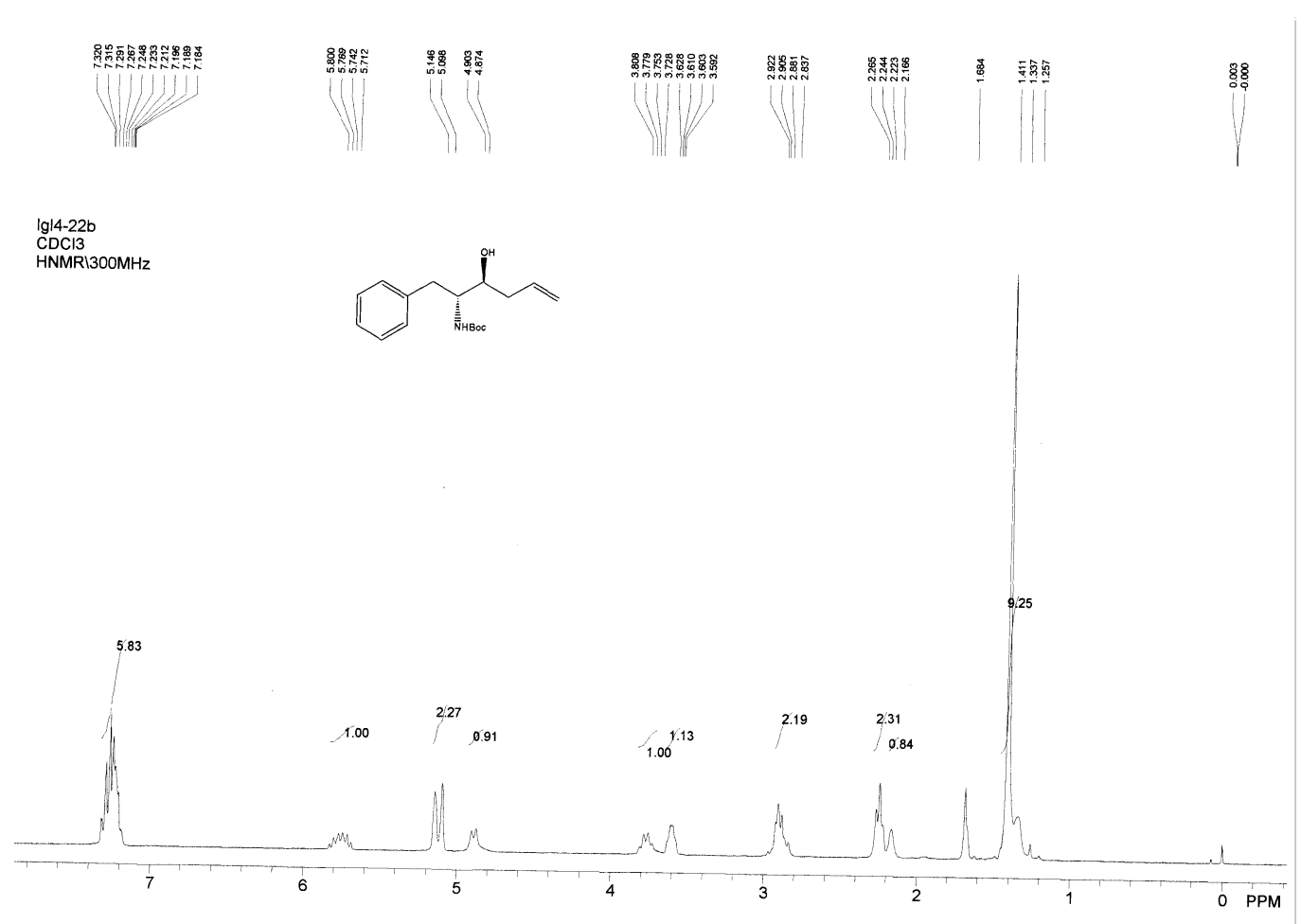

\section{1-phenyl-3-penten-1-ol (6a)}

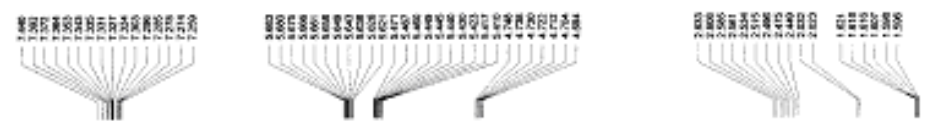

$\operatorname{lgDCl}_{\mathrm{CO}} \mathrm{A}-11 \mathrm{~b}$
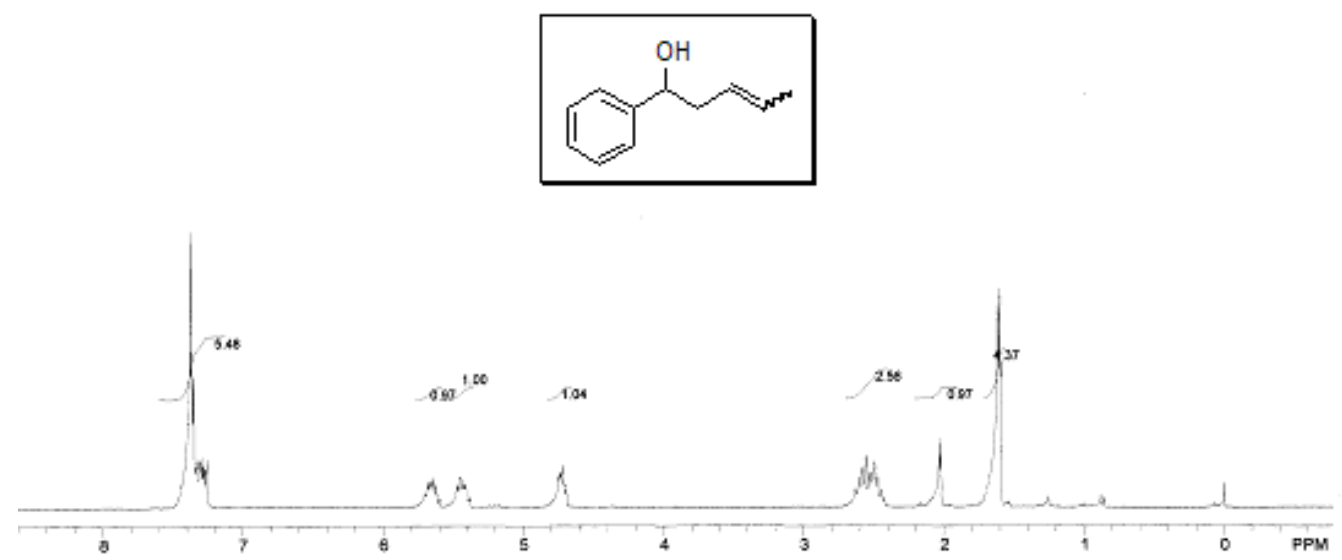
2-methyl-1-phenylbut-3-en-1-ol (7a)

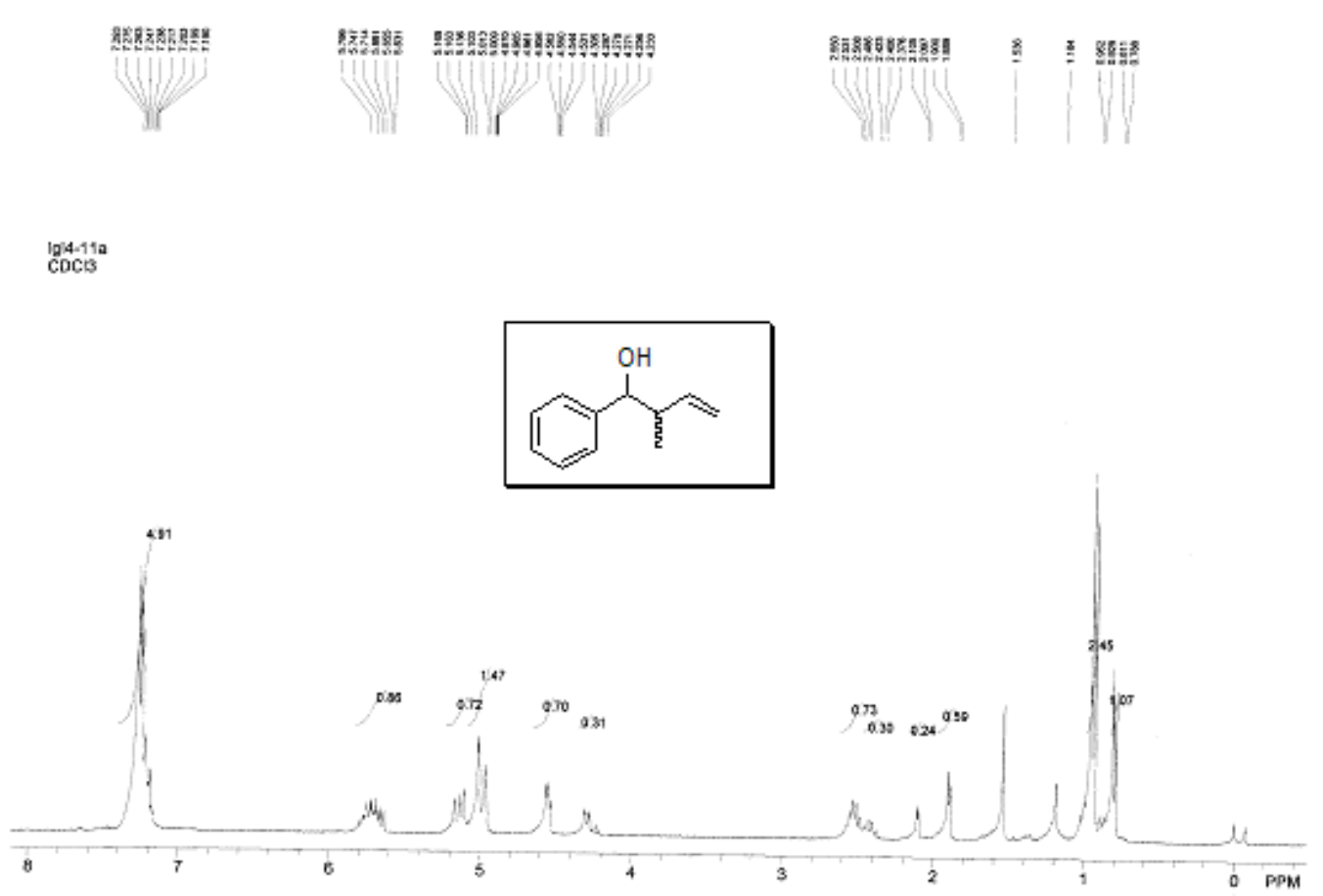

\section{1-(4-nitophenyl)-3-penten-1-ol (6b)}

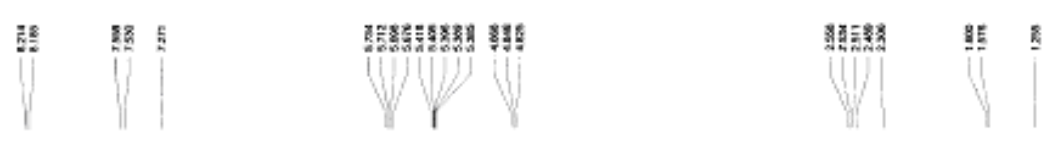

$\underset{c 0 c 3}{9.34 \cdot 2}$
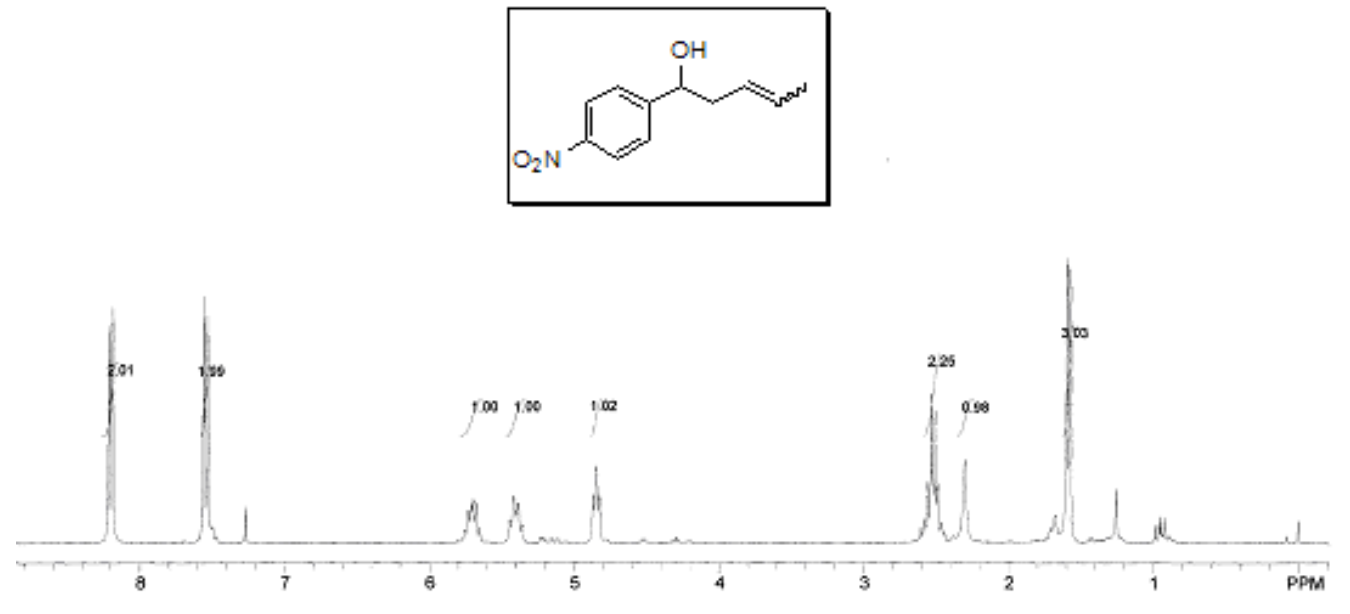
2-methyl-1-(4-nitrophenyl)-3-buten-1-ol (7b)
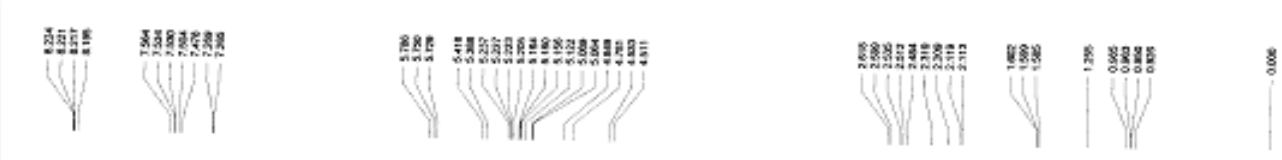

$$
\text { lg/4-34-1 }
$$
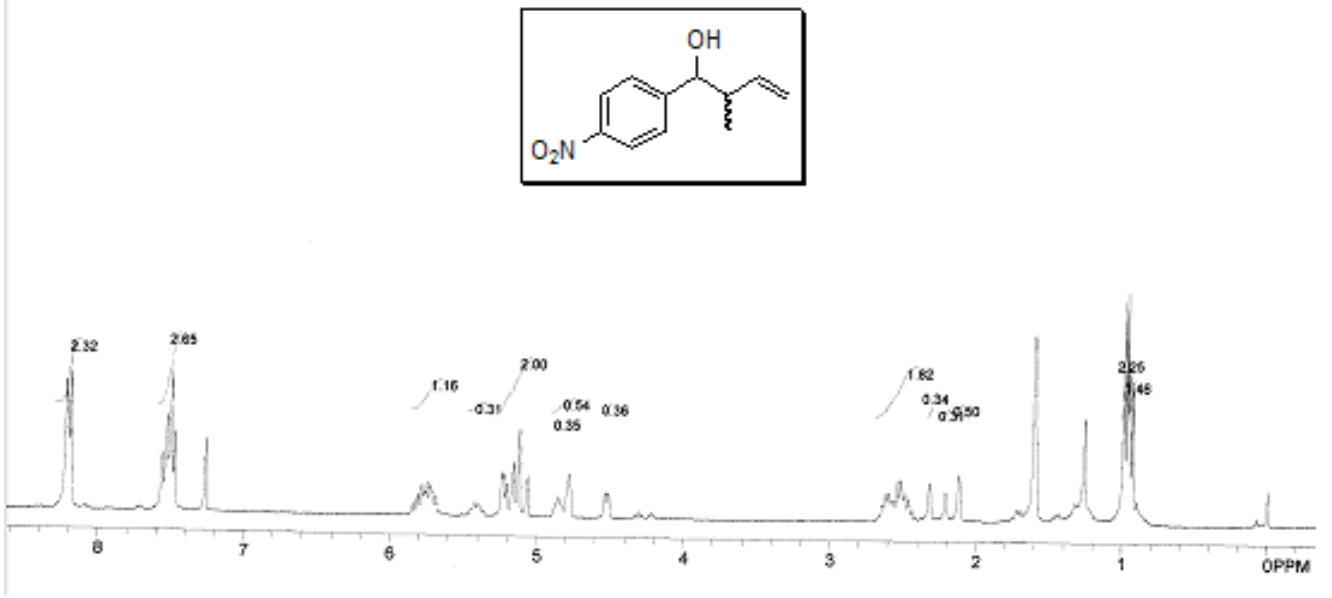

\section{1-(3-methoxyphenyl)-3-penten-1-ol (6c)}

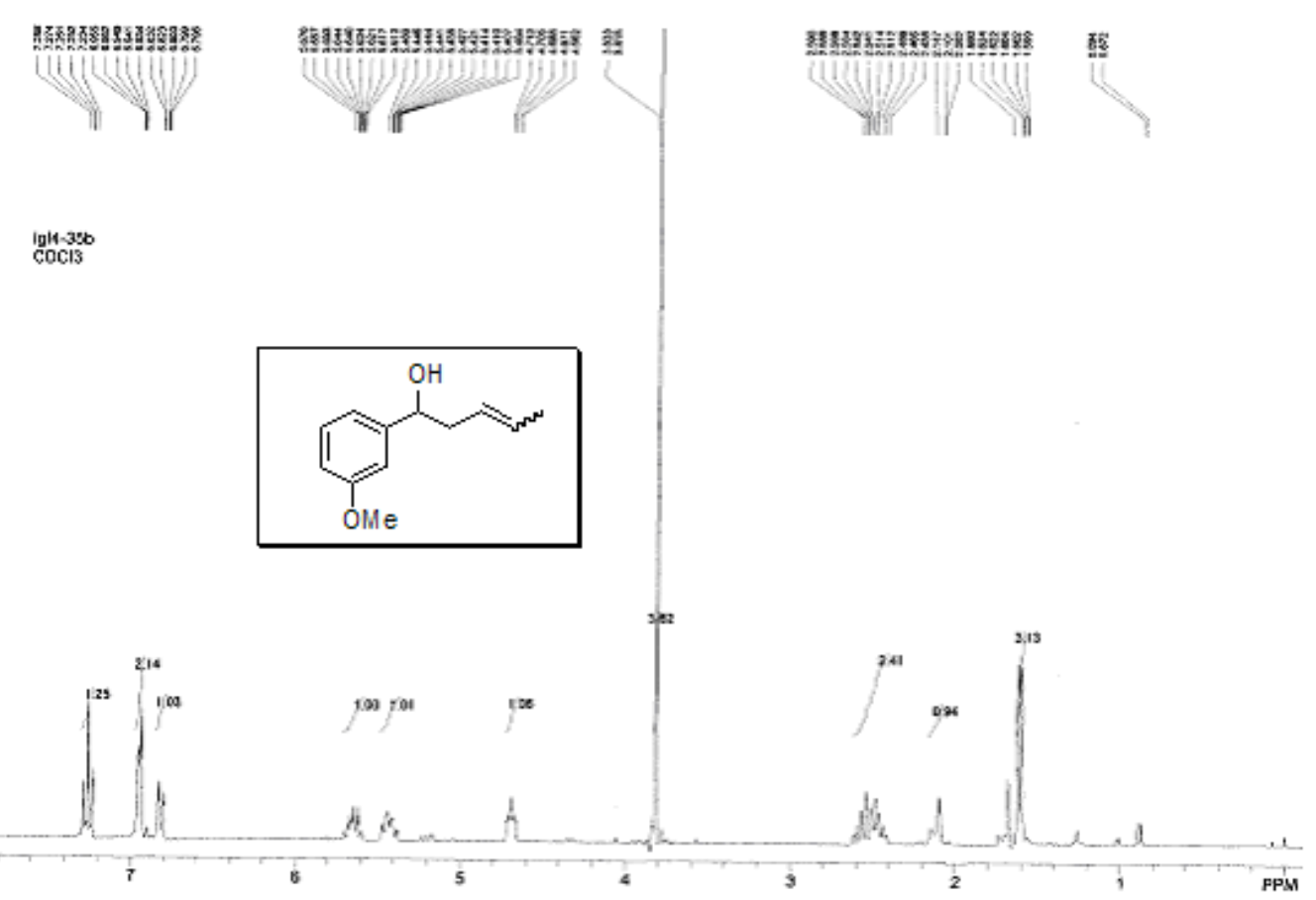


2-methyl-1-(3-methoxyphenyl)-3-buten-1-ol (7c)

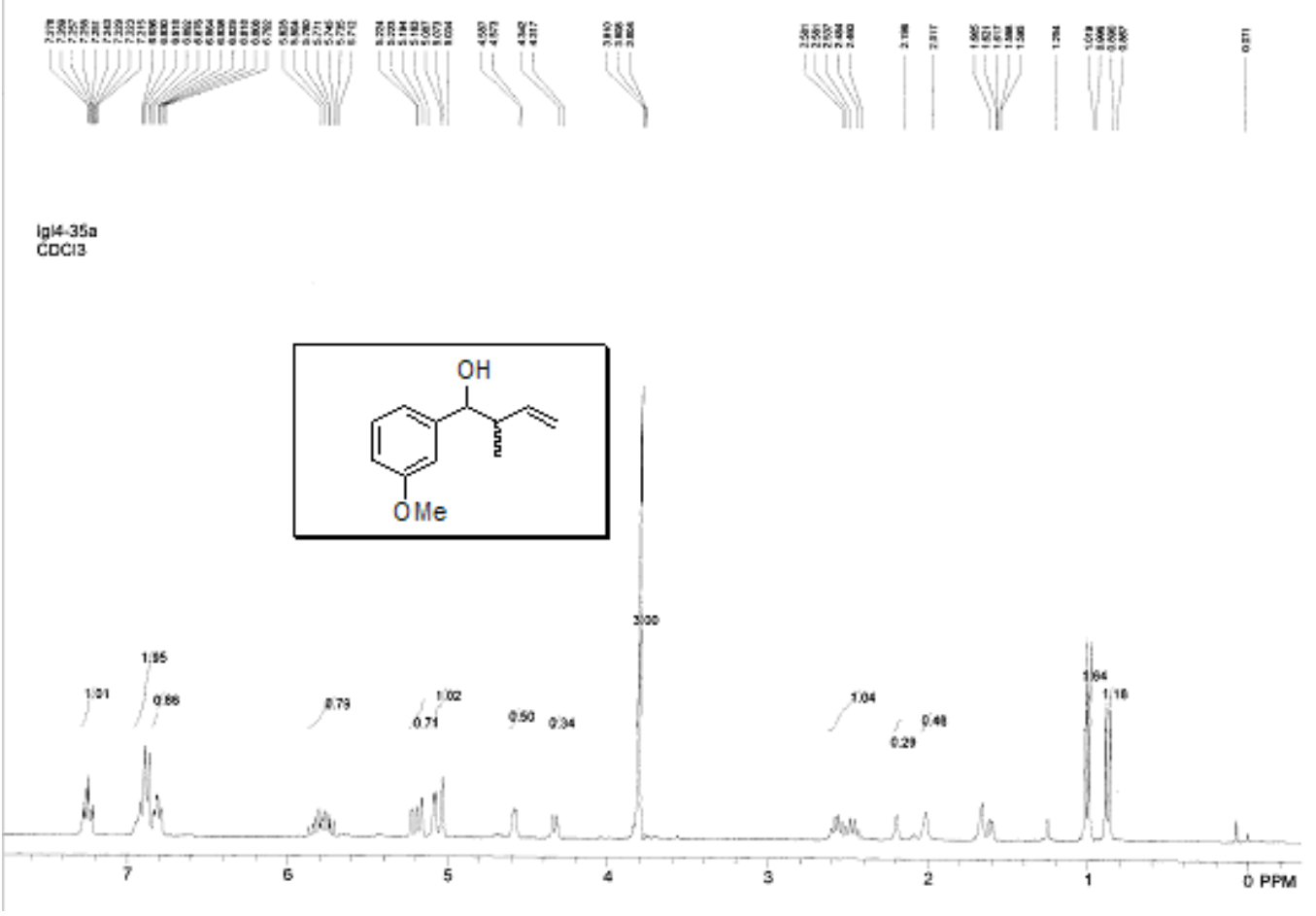

dodec-2-en-5-ol (6d) 


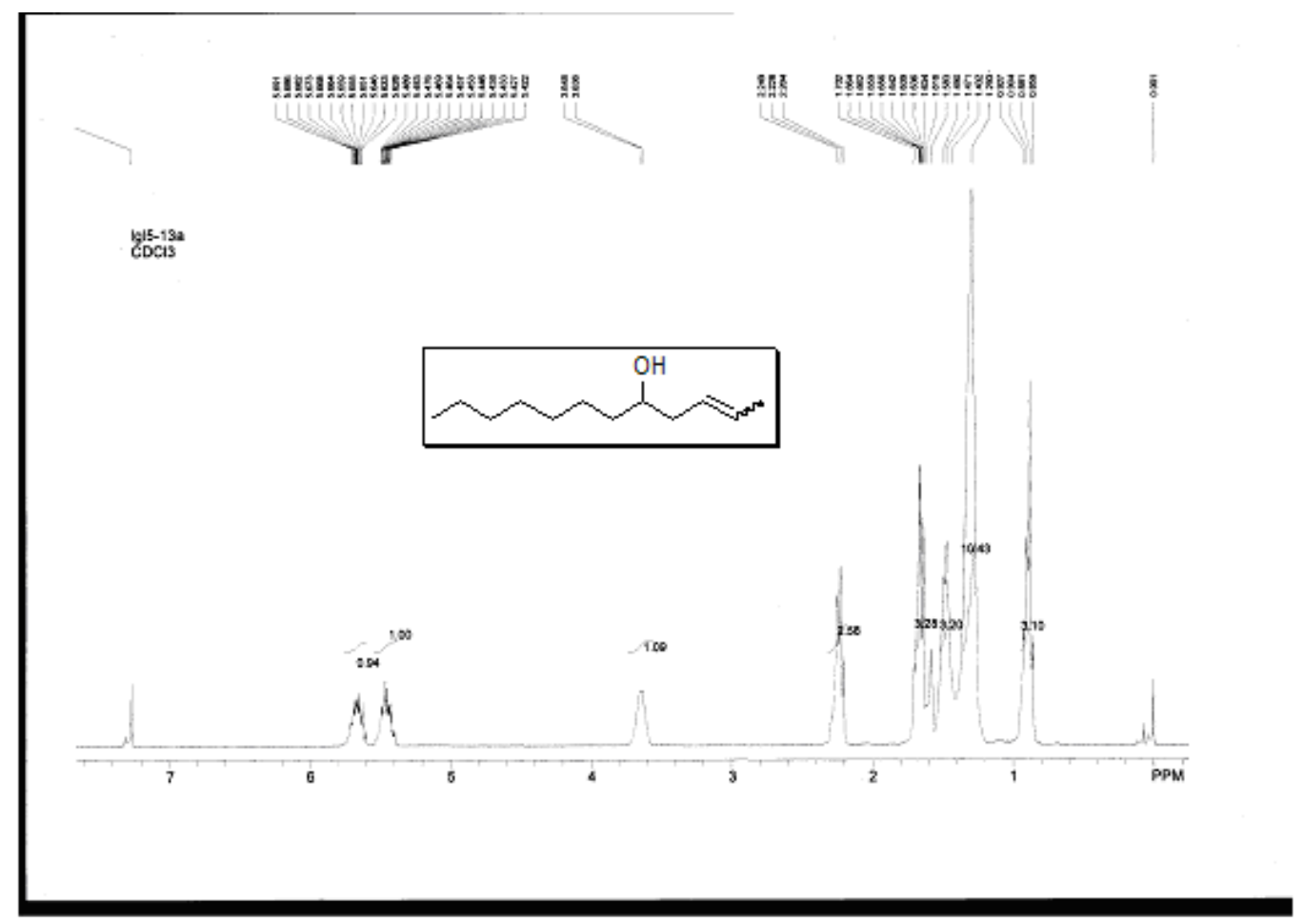

dodec-2-en-5-ol (6d) and 3-methyl-undec-1-en-4ol (7d)

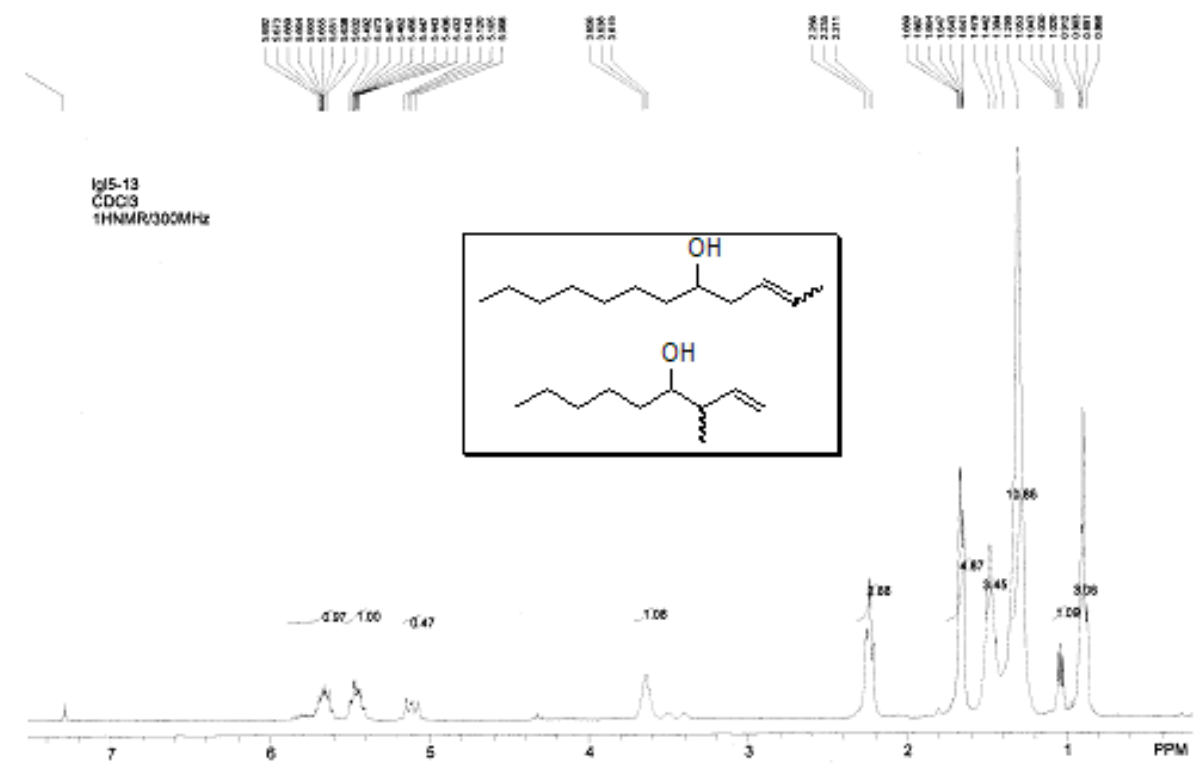




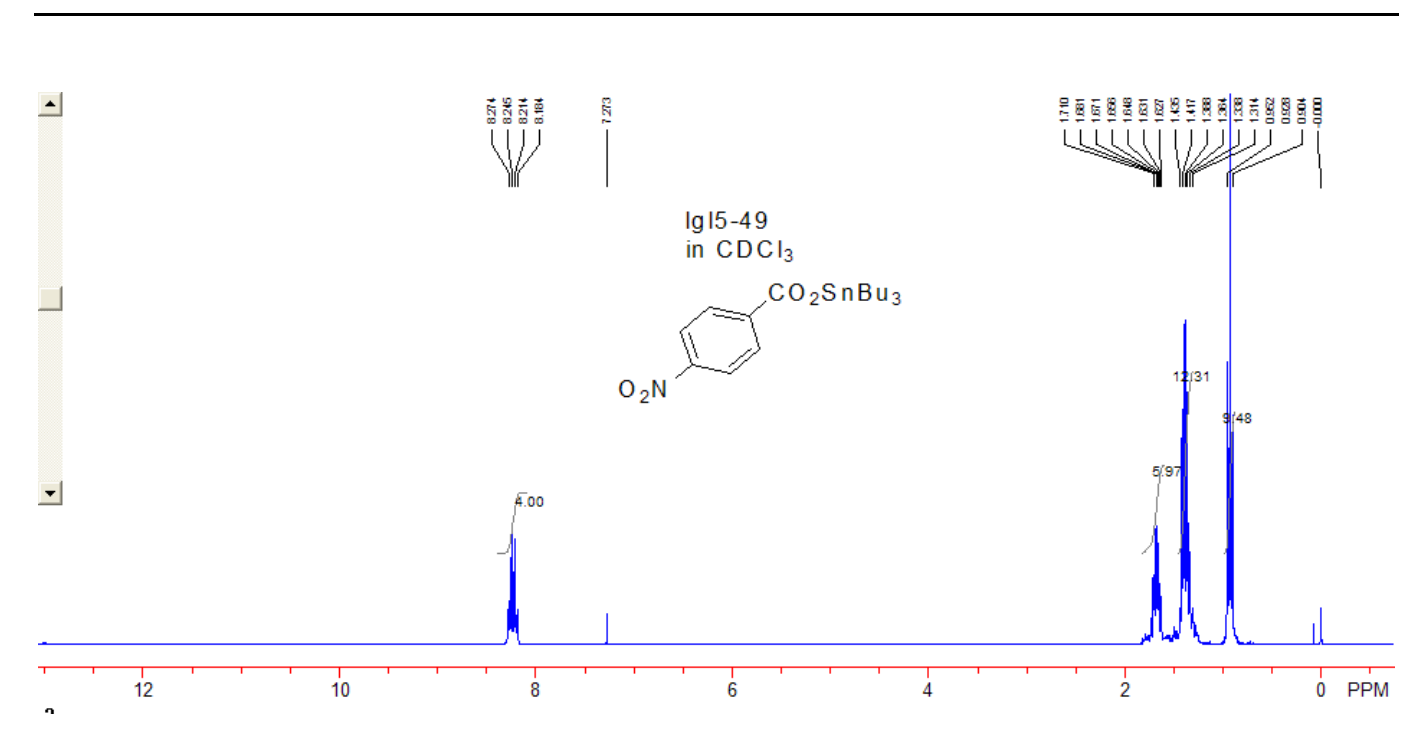

